Chinese Journal of Organic Chemistry

ARTICLE

\title{
3,4-二芳基-5-芳氧甲基异噁唑的合成
}

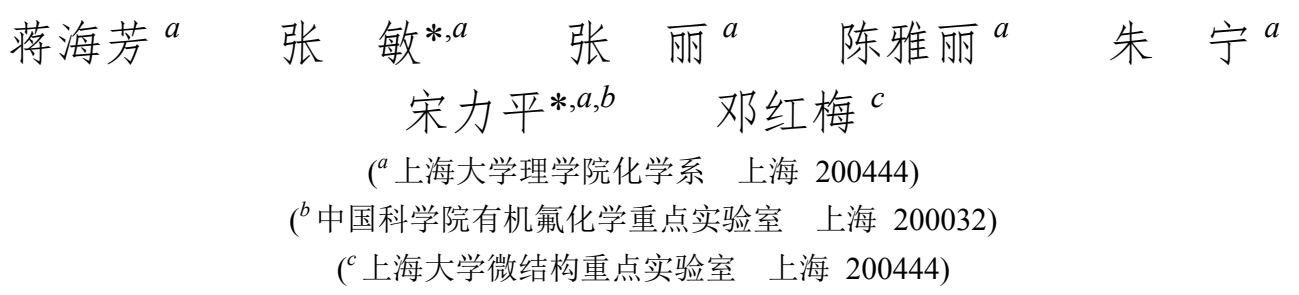

\begin{abstract}
摘要 由于异噁唑衍生物具有广泛的除草、杀虫和药物活性，其合成方法近年来一直引起关注. 以 3-芳基-5-溴甲基异 噁唑为原料, 经溴代、醚化、Suzuki 偶联反应合成了一系列 3,4-二取代芳基-5-芳氧甲基异噁唑化合物，初步的生物活 性测试结果显示，部分化合物对粘虫显示出一定的杀虫活性.

关键词 异惡唑衍生物; 溴代; 醚化; Suzuki 偶联反应
\end{abstract}

\section{Synthesis of 3,4-Diaryl-5-aryloxymethyl Isoxazole Derivatives}

\author{
Jiang, Haifang ${ }^{a} \quad$ Zhang, Min ${ }^{* a} \quad$ Zhang, $\mathrm{Li}^{a} \quad$ Chen Yali $^{a}$ \\ Zhu, Ning ${ }^{a}$ Song, Liping ${ }^{*, a, b}$ Deng, Hongmei ${ }^{c}$ \\ ( ${ }^{a}$ Department of Chemistry, School of Science, Shanghai University, Shanghai 200444) \\ $\left({ }^{b}\right.$ Key Laboratory of Organofluorine Chemistry, Shanghai Institute of Organic Chemistry, \\ Chinese Academy of Sciences, Shanghai 200032) \\ ( ${ }^{c}$ Laboratory for Microstructures, Shanghai University, Shanghai 200444)
}

\begin{abstract}
Due to isoxazole derivatives possess a wide range of herbicidal, insecticidal and pharmacological activitives, the synthetic methodologies for preparation of isoxazole derivatives have drawn much attention in recent years. Herein, 3,4-diaryl-5-aryloxymethyl isoxazole derivatives were effectively synthesized from 3-aryl-5-(bromomethyl)isoxazole via consecutive bromination, etherification, followed by Suzuki coupling reaction catalyzed by $\mathrm{Pd}\left(\mathrm{PPh}_{3}\right)_{2} \mathrm{Cl}_{2}$. Preliminary bioassay data showed that some of the title compounds exhibited certain insecticidal activities against Oriental armyworm.

Keywords isoxazole derivatives; bromination; etherification; suzuki coupling reaction
\end{abstract}

异噁唑类化合物具有非常良好的生物活性，在农 药、医药等领域具有广阔的应用价值 ${ }^{[14]}$. 异噁唑类化 合物不仅被广泛应用于农化产品的研究 ${ }^{[5 \sim 8]}$, 而且在药 物化学 ${ }^{[9]}$ 和材料化学 ${ }^{[10]}$ 上的应用也相当广泛 ${ }^{[11]}$, 可显示 多种药物活性, 如抗菌、抗炎、平喘、降血压、抗肿瘤 等. 因此, 其合成方法也引起了许多药物化学家的注意, 很多异噁唑衍生物已被开发成医药产品 ${ }^{[12 ~ 19]}$. 研究结 果同时发现, 异噁唑衍生物在治疗帕金森氏病和老年痴 呆症方面也有一定的作用 ${ }^{[20,21]}$. 3,4-二芳基异噁唑作为 药物骨架被广泛应用于生物制药, 例如非甾体抗炎药 物、蛋白激酶抑制剂等 ${ }^{[22]}$. 目前, 在异噁唑 4-位上引入
芳基的方法主要可通过 Suzuki 或 Stille 交叉偶联反应来 制备 ${ }^{[22 ~ 31]}$, 但 3,4-二取代芳基-5-芳氧甲基异噁唑化合 物少见报道 ${ }^{[2]}$, 为了进一步研究多取代异噁唑化合物 的构效关系, 本文在前期研究的基础上 ${ }^{[33,34]}$, 以 3 -芳 基-5-溴甲基异噁唑(1)为原料, 经溴代、醚化、Suzuki 偶联反应合成了系列 3,4-二取代芳基-5-芳氧甲基异噁 唑化合物。

\section{1 结果与讨论}

目标产物 3,4-二芳基-5-芳氧甲基异噁唑衍生物的 合成路线如 Scheme 1 所示. 首先, 我们初步尝试了合成

\footnotetext{
* Corresponding authors. E-mail: mzhang@shu.edu.cn; lpsong@shu.edu.cn

Received February 20, 2017; revised April 7, 2017; published online May 24, 2017.

Project supported by the National Natural Science Foundation of China (No. 21272153).

国家自然科学基金(No. 21272153)资助项目.
} 
化合物 2 的溴化条件, 考虑到如果用液溴或 $\mathrm{H}_{2} \mathrm{O}_{2} / \mathrm{HBr}$ 体系进行溴化时反应不如用 $N$-溴代丁二酰亚胺(NBS)溴 化的反应条件温和、容易操作 ${ }^{[35 ~ 37]}$, 所以我们选择了较 为温和的 NBS 作为溴化试剂, 以 3-(4-氰基苯基)-5-溴甲 基异啞唑反应为例, 笁选了不同的溴化反应条件. 当选 择以醋酸为反应溶剂时, 无论是在室温下还是提高反应 温度, 反应均无法进行 (表 1, Entry 2); 当以醋酸/三氟醋 酸 $(V: V=10: 1)$ 为反应溶剂, 反应在 $25{ }^{\circ} \mathrm{C}$ 时亦不能进 行, 只有在 $60{ }^{\circ} \mathrm{C}$ 时才能得到少量目标产物(表 1, Entries $3,4)$; 而当以三氟醋酸为反应溶剂时，该溴化反应能够 顺利进行, 并且在 $60{ }^{\circ} \mathrm{C}$ 下反应 $6 \mathrm{~h}$ 的收率达到 75\% (表 1, Entry 6). 随后我们以此为较优的溴代反应条件, 进一 步考察了以 3-(4-硝基苯基)-5-溴甲基异噁唑、3-(4-甲氧 基苯基)-5-溴甲基异啞唑为底物的溴代反应, 分别以 $83 \%$ 和 $86 \%$ 的产率得到相应的化合物 2 . 化合物 2 在碱 性条件下与取代苯酚发生醚化反应得到化合物 3. 具体 反应条件参见文献[35]方法, 反应产率见表 2. 化合物 3 通过与苯硼酸 4 在 $\mathrm{Pd}\left(\mathrm{PPh}_{3}\right)_{2} \mathrm{Cl}_{2}$ 催化下的 Suzuki 反应得 到一系列的多芳基取代的异啞唑衍生物 $\mathbf{5}$.
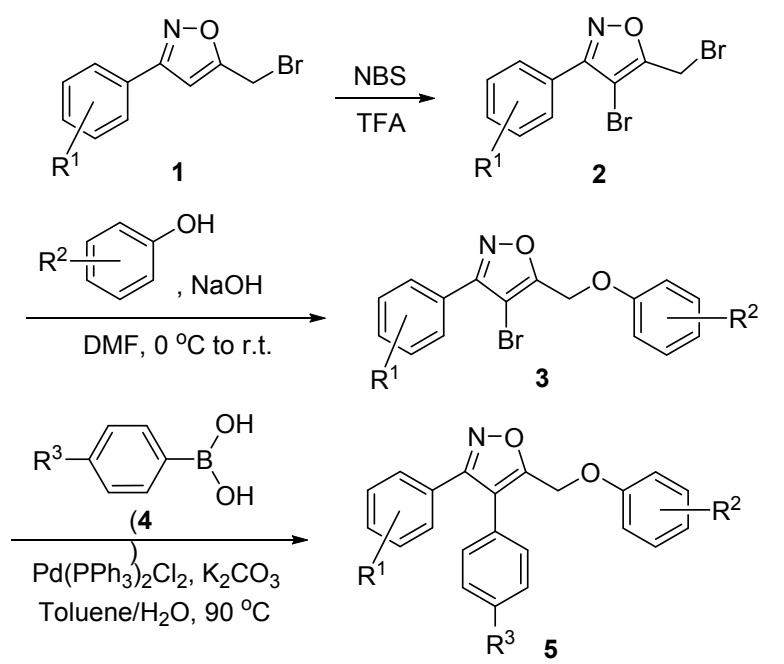

$\mathrm{R}^{1}=p-\mathrm{NO}_{2}, p-\mathrm{CN}, m-\mathrm{OCH}_{3} ; \mathrm{R}^{2}=p-\mathrm{NO}_{2}, m-\mathrm{NO}_{2}, m-\mathrm{CH}_{3}$, $p-\mathrm{CH}_{3} \mathrm{O}, p-\mathrm{F} ; \mathrm{R}^{3}=\mathrm{H}, \mathrm{F}, \mathrm{CF}_{3}$

图式 13,4 -二芳基-5-芳氧甲基异噁唑的合成路线

Scheme 1 Synthetic route for preparation of 3,4-diaryl-5-aryloxy-methyl-isoxazoles

表 1 化合物 2a 的反应条件优化 ${ }^{a}$

Table 1 Optimization of reaction conditions for preparation $\mathbf{2 a}$

\begin{tabular}{|c|c|c|c|c|}
\hline Entry & Solvent & Temp $/{ }^{\circ} \mathrm{C}$ & $T / \mathrm{h}$ & Yield $^{b} / \%$ \\
\hline 1 & DMF & 25 & 12 & $-^{c}$ \\
\hline 2 & $\mathrm{AcOH}$ & 60 & 12 & $-^{c}$ \\
\hline 3 & $\operatorname{AcOH} / \mathrm{TFA}(V / V=10 / 1)$ & 25 & 12 & $-^{c}$ \\
\hline 4 & $\operatorname{AcOH} / \mathrm{TFA}(V / V=10 / 1)$ & 60 & 12 & 25 \\
\hline 5 & TFA & 25 & 12 & 37 \\
\hline 6 & TFA & 60 & 6 & 75 \\
\hline
\end{tabular}

表 2 异噁唑衍生物 3-1 3-10 的合成 ${ }^{a}$

Table 2 Synthesis of isoxazole derivatives 3-1 $\sim$ 3-10

\begin{tabular}{cllcc}
\hline Entry & \multicolumn{1}{c}{$\mathrm{R}^{1}$} & \multicolumn{1}{c}{$\mathrm{R}^{2}$} & Product & Yield $^{b} \%$ \\
\hline 1 & $4-\mathrm{CN}$ & $3-\mathrm{NO}_{2}$ & $\mathbf{3 - 1}$ & 87 \\
2 & $4-\mathrm{CN}$ & $4-\mathrm{CH}_{3} \mathrm{O}$ & $\mathbf{3 - 2}$ & 85 \\
3 & $4-\mathrm{CN}$ & $4-\mathrm{F}$ & $\mathbf{3 - 3}$ & 84 \\
4 & $4-\mathrm{NO}$, & $4-\mathrm{CH}_{3} \mathrm{O}$ & $\mathbf{3 - 4}$ & 88 \\
5 & $4-\mathrm{NO}_{2}$ & $3-\mathrm{NO}_{2}$ & $\mathbf{3 - 5}$ & 69 \\
6 & $4-\mathrm{NO}_{2}$ & $3-\mathrm{Me}$ & $\mathbf{3 - 6}$ & 85 \\
7 & $4-\mathrm{CH}_{3} \mathrm{O}$ & $4-\mathrm{CH}_{3} \mathrm{O}$ & $\mathbf{3 - 7}$ & 90 \\
8 & $4-\mathrm{CH}_{3} \mathrm{O}$ & $3-\mathrm{NO}_{2}$ & $\mathbf{3 - 8}$ & 75 \\
9 & $4-\mathrm{CH}_{3} \mathrm{O}$ & $4-\mathrm{F}$ & $\mathbf{3 - 9}$ & 86 \\
10 & $4-\mathrm{CH}_{3} \mathrm{O}$ & $3-\mathrm{Me}^{3}$ & $\mathbf{3 - 1 0}$ & 88 \\
\hline${ }^{a}{ }^{\circ}$ & &
\end{tabular}

${ }^{a}$ Reaction conditions: $25{ }^{\circ} \mathrm{C}, 12 \mathrm{~h} ;{ }^{b}$ Isolated yield.

由表 3 结果可见, 取代基的电子效应对目标产物产 率的影响不是很明显, 该反应的普适性较好. 对目标产 物的结构鉴定, 不仅通过 ${ }^{1} \mathrm{H} N \mathrm{NMR} 、{ }^{13} \mathrm{C} N \mathrm{~N}$ 、 IR 和 HRMS 进行了表征，同时还对代表性的化合物 5 16 进 行了单晶培养, 并对所得晶体进行了 X-射线晶体结构 分析 ${ }^{[38]}$. 晶体结构见图 1, 进一步确定了化合物的结构.

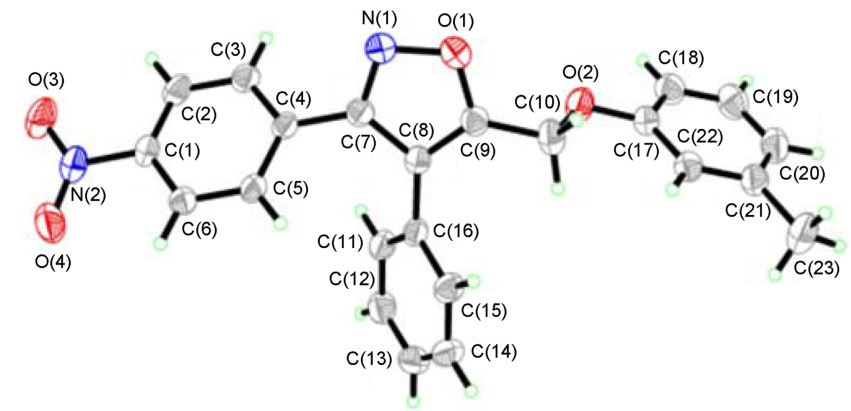

图 1 化合物 5-16 的晶体结构

Figure 1 X-ray crystal structure of 5-16

化合物 5-16 的晶体结构属正交晶系，四个芳环都 不在一个平面上，其中异噁唑环与对硝基苯环的两面角 为 $43.3^{\circ}$, 异噁唑环与苯环的两面角为 $50.1^{\circ}$, 异噁唑环 与 3-甲基苯环的两面角为 $-73.0^{\circ}$.

我们对目标化合物进行了昆虫室内毒力测试, 供试 对象是豆蚜 Aphis crassivora 成蚜和东方粘虫 M. separata 2 龄幼虫. 发现目标化合物对豆蚜 Aphis crassivora 成蚜无活性，对东方粘虫 M. separata 2 龄幼虫发现测试 三天后目标化合物 5-19 的杀虫率为 23.3\%, 5-24 的杀虫 率为 $26.7 \%$, 其余样品杀虫率较低, 仅为 $0 \% \sim 13.3 \%$.

\section{2 结论}

以 3-芳基-5-溴甲基异噁唑(1)为原料，经溴代、醚 化、Suzuki 偶联反应高效地合成了一系列 3,4-二取代芳 基-5-芳氧甲基异噁唑化合物 5, 并测试了化合物 5-16 的 单晶结构, 为进一步研究多芳基取代的异噁唑化合物的 
表 3 异噁唑衍生物 5-1 5-30 的合成 ${ }^{a}$

Table 3 Synthesis of isoxazole derivatives 5-1 $\sim$ 5-30

\begin{tabular}{|c|c|c|c|c|c|}
\hline Entry & $\mathrm{R}^{1}$ & $\mathrm{R}^{2}$ & $\mathrm{R}^{3}$ & Product & Yield $^{b} / \%$ \\
\hline 1 & $4-\mathrm{CN}$ & $4-\mathrm{NO}_{2}$ & $\mathrm{H}$ & $5-1$ & 88 \\
\hline 2 & $4-\mathrm{CN}$ & $4-\mathrm{NO}_{2}$ & $\mathrm{~F}$ & $5-2$ & 86 \\
\hline 3 & $4-\mathrm{CN}$ & $3-\mathrm{NO}_{2}$ & $4-\mathrm{CF}_{3}$ & $5-3$ & 91 \\
\hline 4 & $4-\mathrm{CN}$ & 4- $\mathrm{CH}_{3} \mathrm{O}$ & $\mathrm{H}$ & $5-4$ & 82 \\
\hline 5 & $4-\mathrm{CN}$ & $4-\mathrm{CH}_{3} \mathrm{O}$ & $4-\mathrm{F}$ & $5-5$ & 91 \\
\hline 6 & $4-\mathrm{CN}$ & $4-\mathrm{CH}_{3} \mathrm{O}$ & $4-\mathrm{CF}_{3}$ & $5-6$ & 92 \\
\hline 7 & $4-\mathrm{CN}$ & $4-\mathrm{F}$ & $\mathrm{H}$ & $5-7$ & 89 \\
\hline 8 & $4-\mathrm{CN}$ & $4-\mathrm{F}$ & $4-\mathrm{F}$ & $5-8$ & 82 \\
\hline 9 & $4-\mathrm{CN}$ & $4-\mathrm{F}$ & $4-\mathrm{CF}_{3}$ & $5-9$ & 85 \\
\hline 10 & $4-\mathrm{NO}_{2}$ & 4- $\mathrm{CH}_{3} \mathrm{O}$ & $\mathrm{H}$ & $5-10$ & 85 \\
\hline 11 & $4-\mathrm{NO}_{2}$ & 4- $\mathrm{CH}_{3} \mathrm{O}$ & $4-F$ & $5-11$ & 83 \\
\hline 12 & $4-\mathrm{NO}_{2}$ & 4- $\mathrm{CH}_{3} \mathrm{O}$ & $4-\mathrm{CF}_{3}$ & $5-12$ & 86 \\
\hline 13 & $4-\mathrm{NO}_{2}$ & $3-\mathrm{NO}_{2}$ & $\mathrm{H}$ & $5-13$ & 84 \\
\hline 14 & $4-\mathrm{NO}_{2}$ & $3-\mathrm{NO}_{2}$ & $4-F$ & $5-14$ & 81 \\
\hline 15 & $4-\mathrm{NO}_{2}$ & $3-\mathrm{NO}_{2}$ & $4-\mathrm{CF}_{3}$ & $5-15$ & 84 \\
\hline 16 & $4-\mathrm{NO}_{2}$ & 3-Me & $\mathrm{H}$ & $5-16$ & 83 \\
\hline 17 & $4-\mathrm{NO}_{2}$ & 3-Me & $4-\mathrm{F}$ & $5-17$ & 83 \\
\hline 18 & $4-\mathrm{NO}_{2}$ & 3-Me & $4-\mathrm{CF}_{3}$ & $5-18$ & 85 \\
\hline 19 & $3-\mathrm{CH}_{3} \mathrm{O}$ & 4- $\mathrm{CH}_{3} \mathrm{O}$ & $\mathrm{H}$ & $5-19$ & 89 \\
\hline 20 & $3-\mathrm{CH}_{3} \mathrm{O}$ & 4- $\mathrm{CH}_{3} \mathrm{O}$ & $4-F$ & $5-20$ & 91 \\
\hline 21 & $3-\mathrm{CH}_{3} \mathrm{O}$ & 4- $\mathrm{CH}_{3} \mathrm{O}$ & $4-\mathrm{CF}_{3}$ & $5-21$ & 88 \\
\hline 22 & $3-\mathrm{CH}_{3} \mathrm{O}$ & $3-\mathrm{NO}_{2}$ & $\mathrm{H}$ & $5-22$ & 85 \\
\hline 23 & $3-\mathrm{CH}_{3} \mathrm{O}$ & $3-\mathrm{NO}_{2}$ & $4-F$ & $5-23$ & 85 \\
\hline 24 & $3-\mathrm{CH}_{3} \mathrm{O}$ & $3-\mathrm{NO}_{2}$ & $4-\mathrm{CF}_{3}$ & $5-24$ & 82 \\
\hline 25 & $3-\mathrm{CH}_{3} \mathrm{O}$ & $4-\mathrm{F}$ & $\mathrm{H}$ & $5-25$ & 90 \\
\hline 26 & $3-\mathrm{CH}_{3} \mathrm{O}$ & $4-\mathrm{F}$ & $4-\mathrm{F}$ & $5-26$ & 88 \\
\hline 27 & $3-\mathrm{CH}_{3} \mathrm{O}$ & $4-\mathrm{F}$ & $4-\mathrm{CF}_{3}$ & $5-27$ & 82 \\
\hline 28 & $3-\mathrm{CH}_{3} \mathrm{O}$ & $3-\mathrm{Me}$ & $\mathrm{H}$ & $5-28$ & 91 \\
\hline 29 & $3-\mathrm{CH}_{3} \mathrm{O}$ & 3-Me & $4-F$ & $5-29$ & 84 \\
\hline 30 & $3-\mathrm{CH}_{3} \mathrm{O}$ & $3-\mathrm{Me}$ & $4-\mathrm{CF}_{3}$ & $5-30$ & 86 \\
\hline
\end{tabular}

${ }^{a}$ Reaction conditions: $90{ }^{\circ} \mathrm{C}, 12 \mathrm{~h} .{ }^{b}$ Isolated yield.

生物活性和构效关系奠定了基础.

\section{3 实验部分}

\section{1 仪器和试剂}

Brucker AV-500 核磁共振光谱仪, TMS 内标, $\mathrm{CDCl}_{3}$ 或 DMSO- $d_{6}$ 作为溶剂; LCMS 2020 低分辨质谱仪; Bruker Daltonics APEXIII 7.0 TESALA FTMS 高分辨质 谱仪; WRS-1A 数字熔点仪; 柱层析硅胶为 $\mathrm{H}$ 型(青岛海 洋化工厂); 所用试剂均为市售分析, 用前未作处理. 3芳基-5-澳甲基异噁唑 (1) 均从 APICHEMICAL SHANGHAI CO. LTD 公司购买.

\section{2 实验方法}

\section{2 .1 化合物 $\mathbf{2}$ 的合成}

称取 4-氧基苯基-5-溴甲基异噁唑(1) (1.0 g, 3.58 $\mathrm{mmol}$ )于 $50 \mathrm{~mL}$ 圆底烧瓶中, 加入 $20 \mathrm{~mL}$ 三氟醋酸, 室 温搅拌至完全溶解后加入 NBS (0.96 g, $7.19 \mathrm{mmol})$, $60^{\circ} \mathrm{C}$ 下反应约 6 个 $\mathrm{h}$, 薄层色谱(TLC)检测至反应完全.
混合物冷却后倒入盛有 $10 \mathrm{~mL}$ 水的 $100 \mathrm{~mL}$ 烧杯中, 用 $\mathrm{Na}_{2} \mathrm{CO}_{3}$ 调 $\mathrm{pH}$ 值至中性后用乙酸乙酯萃取, 萃取液用无 水 $\mathrm{MgSO}_{4}$ 干燥后浓缩得粗产物, 粗产物用 $V$ ( 乙酸乙 酯)：V(石油醚 $)=1: 10$ 为淋洗剂, 经柱层析分离得到 4-氧基苯基-4-澳-5-溴甲基异噁唑(2-1) $910 \mathrm{mg}$, 产率为 $75 \%$. 化合物 2-2 2-3 按上述方法合成.

3-(4-㲵基苯基)-4-溴-5-溴甲基异噁唑(2-1): 白色固 体, 产率 65.2\%. m.p. 112.5 113.1 ${ }^{\circ} \mathrm{C} ;{ }^{1} \mathrm{H}$ NMR (500 $\left.\mathrm{MHz}, \mathrm{CDCl}_{3}\right) \delta: 7.93(\mathrm{dd}, J=6.5,2.0 \mathrm{~Hz}, 2 \mathrm{H}), 7.77(\mathrm{dd}$, $J=6.5,2.0 \mathrm{~Hz}, 2 \mathrm{H}), 4.54$ (s, 2H).

3-(4-硝基苯基)-4-溴-5-溴甲基异噁唑(2-2): 白色固 体, 产率 74.3\%. m.p. 121.3 122.9 ${ }^{\circ} \mathrm{C}$; ${ }^{1} \mathrm{H}$ NMR (500 $\left.\mathrm{MHz}, \mathrm{CDCl}_{3}\right) \delta: 8.37(\mathrm{dd}, J=9.0,2.0 \mathrm{~Hz}, 2 \mathrm{H}), 8.09(\mathrm{dd}$, $J=9.0,2.0 \mathrm{~Hz}, 2 \mathrm{H}), 4.72(\mathrm{~s}, 0.9 \mathrm{H}), 4.56(\mathrm{~s}, 1.1 \mathrm{H})$.

3-(3-甲氧基苯基)-4-溴-5-溴甲基异噁唑(2-3): 白色 固体, 产率 57.8\%. m.p. 104.7 105.9 ${ }^{\circ} \mathrm{C} ;{ }^{1} \mathrm{H}$ NMR (500 $\left.\mathrm{MHz}, \mathrm{CDCl}_{3}\right) \delta: 7.93(\mathrm{t}, J=2.0 \mathrm{~Hz}, 1 \mathrm{H}), 7.72(\mathrm{~d}, J=9.0$ $\mathrm{Hz}, 1 \mathrm{H}), 6.96$ (d, $J=9.0 \mathrm{~Hz}, 1 \mathrm{H}), 6.57$ (s, 1H), 4.65 (s, 0.7 $\mathrm{H}), 4.50(\mathrm{~s}, 1.4 \mathrm{H}), 3.95(\mathrm{~s}, 3 \mathrm{H})$.

\section{2 .2 化合物 $\mathbf{3}$ 的合成}

称取 3-硝基苯酚 $(0.19 \mathrm{~g}, 1.39 \mathrm{mmol})$ 于 $25 \mathrm{~mL}$ 圆底 烧瓶中, 加入 $10 \mathrm{~mL} N, N$-二甲基甲酰胺(DMF)溶解, 室 温条件下加入 $\mathrm{NaOH}(0.06 \mathrm{~g}, 1.52 \mathrm{mmol})$, 反应 $1 \mathrm{~h}$ 后加 入 4-㲵基苯基-4-溴-5-溴甲基异噁唑(2) $(0.5 \mathrm{~g}, 1.39$ $\mathrm{mmol}$ ), 室温下反应 $12 \mathrm{~h}, \mathrm{TLC}$ 检测反应完全. 将反应液 倒入 $30 \mathrm{~mL}$ 水中, 混合液用乙酸乙酯 $(30 \mathrm{~mL} \times 3)$ 萃取, 收集有机相, 用无水 $\mathrm{MgSO}_{4}$ 干燥后浓缩得粗产物, 粗产 物用 $V$ (乙酸乙酯) $: V$ (石油醚 $)=1: 8$ 为淋洗剂, 经柱层 析分离得 3-(4-氧基苯基)-4-溴-5-(3-硝基苯氧甲基)异噁 坐(3-1) $480 \mathrm{mg}$, 产率为 87\%. 化合物 3-2 3-10 按上述 方法合成.

3-(4-氧基苯基)-4-澳-5-(3-硝基苯氧甲基)异噁唑 (3-1): 白色固体, 产率 86.1\%. m.p. $149.4 \sim 149.8{ }^{\circ} \mathrm{C} ;{ }^{1} \mathrm{H}$ NMR $\left(500 \mathrm{MHz}, \mathrm{CDCl}_{3}\right) \delta: 8.02 \sim 8.00(\mathrm{~m}, 2 \mathrm{H}), 7.94 \sim$ $7.92(\mathrm{~m}, 1 \mathrm{H}), 7.89(\mathrm{t}, J=2.5 \mathrm{~Hz}, 1 \mathrm{H}), 7.82 \sim 7.80(\mathrm{~m}, 2 \mathrm{H})$, $7.51(\mathrm{t}, J=8.0 \mathrm{~Hz}, 1 \mathrm{H}), 7.37 \sim 7.34(\mathrm{~m}, 1 \mathrm{H}), 5.32(\mathrm{~s}, 2 \mathrm{H})$; ${ }^{13} \mathrm{C}$ NMR $\left(126 \mathrm{MHz}, \mathrm{CDCl}_{3}\right) \delta: 165.15,159.39,158.04$, $149.23,132.58,131.55,130.45,128.85,121.96,118.11$, $117.23,114.34,109.32,94.08,59.91$.

3-(4-氧基苯基)-4-澳-5-(4-甲氧基苯氧甲基)异噁坐 (3-2): 白色固体, 产率 83.7\%. m.p. $110.8 \sim 111.3{ }^{\circ} \mathrm{C} ;{ }^{1} \mathrm{H}$ NMR $\left(500 \mathrm{MHz}, \mathrm{CDCl}_{3}\right) \delta: 8.02 \sim 8.00(\mathrm{~m}, 2 \mathrm{H}), 7.81 \sim$ $7.79(\mathrm{~m}, 2 \mathrm{H}), 6.97 \sim 6.95(\mathrm{~m}, 2 \mathrm{H}), 5.17(\mathrm{~s}, 2 \mathrm{H}), 3.78(\mathrm{~s}$, $3 \mathrm{H}) ;{ }^{13} \mathrm{C}$ NMR $\left(126 \mathrm{MHz}, \mathrm{CDCl}_{3}\right) \delta: 166.58,159.21$, $154.97,151.77,132.52,131.85,128.85,118.17,116.44$, 
114.81, 114.18, 93.34, 60.69, 55.71.

3-(4-氰基苯基)-4-溴-5-(4-氟苯氧甲基)异啞唑(3-3): 白色固体, 产率 79.9\%. m.p. 106.5 106.7 ${ }^{\circ} \mathrm{C} ;{ }^{1} \mathrm{H}$ NMR $\left(500 \mathrm{MHz}, \mathrm{CDCl}_{3}\right) \delta: 8.00(\mathrm{~d}, J=8.0 \mathrm{~Hz}, 2 \mathrm{H}), 7.79$ (d, $J=$ $8.0 \mathrm{~Hz}, 2 \mathrm{H}), 7.10 \sim 6.88(\mathrm{~m}, 4 \mathrm{H}), 5.18(\mathrm{~s}, 2 \mathrm{H}) ;{ }^{19} \mathrm{~F}$ NMR $\left(471 \mathrm{MHz}, \mathrm{CDCl}_{3}\right) \delta:-121.61 \sim-121.64(\mathrm{~m}) ;{ }^{13} \mathrm{C} \mathrm{NMR}$ $\left(126 \mathrm{MHz}, \mathrm{CDCl}_{3}\right) \delta: 166.17,159.20,158.04\left(\mathrm{~d},{ }^{1} J_{\mathrm{C}-\mathrm{F}}=\right.$ $240.8 \mathrm{~Hz}, \mathrm{ArF}), 153.79,132.53,131.70,128.80,118.18$, $116.44,\left(\mathrm{~d},{ }^{3} J_{\mathrm{C}-\mathrm{F}}=8.1 \mathrm{~Hz}, \operatorname{ArF}\right) 116.17\left(\mathrm{~d},{ }^{2} J_{\mathrm{C}^{-} \mathrm{F}}=23.2 \mathrm{~Hz}\right.$, ArF), 114.17, 93.50, 60.44.

3-(4-硝基苯基)-4-溴-5-(4-甲氧基苯氧甲基)异噁唑 (3-4): 白色固体, 产率 85.2\%. m.p. 104.1 104.7 ${ }^{\circ} \mathrm{C} ;{ }^{1} \mathrm{H}$ NMR $\left(500 \mathrm{MHz}, \mathrm{CDCl}_{3}\right) \delta: 9.09 \sim 8.19(\mathrm{~m}, 2 \mathrm{H}), 8.18 \sim$ $7.98(\mathrm{~m}, 2 \mathrm{H}), 7.07 \sim 6.92(\mathrm{~m}, 2 \mathrm{H}), 6.94 \sim 6.80(\mathrm{~m}, 2 \mathrm{H})$, $5.18(\mathrm{~s}, 2 \mathrm{H}), 3.78(\mathrm{~s}, 3 \mathrm{H}) ;{ }^{13} \mathrm{C} \mathrm{NMR}\left(126 \mathrm{MHz}, \mathrm{CDCl}_{3}\right) \delta$ : $166.70,159.01,154.98,151.76,148.93,133.59,129.24$, 123.95, 116.44, 114.82, 93.41, 60.69, 55.70.

3-(4-硝基苯基)-4-溴-5-(3-硝基苯氧甲基)异噁唑 (3-5): 白色固体, 产率 93.1\%, m.p. 148.7 149.1 ${ }^{\circ} \mathrm{C} ;{ }^{1} \mathrm{H}$ NMR $\left(500 \mathrm{MHz}, \mathrm{CDCl}_{3}\right) \delta: 8.38 \sim 8.36(\mathrm{~m}, 2 \mathrm{H}), 8.10 \sim$ $8.07(\mathrm{~m}, 2 \mathrm{H}), 7.90(\mathrm{t}, J=2.5 \mathrm{~Hz}, 1 \mathrm{H}), 7.51(\mathrm{t}, J=8.0 \mathrm{~Hz}$, $1 \mathrm{H}), 7.49 \sim 7.37(\mathrm{~m}, 1 \mathrm{H}), 5.33(\mathrm{~s}, 2 \mathrm{H}) ;{ }^{13} \mathrm{C} \mathrm{NMR}(126$ $\left.\mathrm{MHz}, \mathrm{CDCl}_{3}\right) \delta: 165.27,159.18,158.04,149.23,149.02$, $133.29,130.46,129.26,124.00,121.96,117.24,109.31$, $94.14,59.90$.

3-(4-硝基苯基)-4-溴-5-((3-甲基苯氧甲基)异啞唑 (3-6): 白色固体, 产率 89.3\%. m.p. 95.8 96.3 ${ }^{\circ} \mathrm{C} ;{ }^{1} \mathrm{H}$ NMR (500 MHz, $\left.\mathrm{CDCl}_{3}\right) \delta: 8.36(\mathrm{~d}, J=9.0 \mathrm{~Hz}, 2 \mathrm{H}), 8.09$ $(\mathrm{d}, J=9.0 \mathrm{~Hz}, 2 \mathrm{H}), 7.22(\mathrm{t}, J=8.0 \mathrm{~Hz}, 1 \mathrm{H}), 6.87 \sim 6.82$ $(\mathrm{m}, 3 \mathrm{H}), 5.22(\mathrm{~s}, 2 \mathrm{H}), 2.36(\mathrm{~s}, 3 \mathrm{H}) ;{ }^{13} \mathrm{C} \mathrm{NMR}(126 \mathrm{MHz}$, $\left.\mathrm{CDCl}_{3}\right) \delta: 166.58,159.03,157.70,148.93,139.94,133.59$, $129.48,129.25,123.95,123.05,115.80,111.68,93.45$, $59.55,21.54$.

3-(3-甲氧基苯基)-4-溴-5-(4-甲氧基苯氧甲基)异噁 唑(3-7): 白色固体, 产率 91.6\%. m.p. 116.5 117.1 ${ }^{\circ} \mathrm{C}$; ${ }^{1} \mathrm{H}$ NMR $\left(500 \mathrm{MHz}, \mathrm{CDCl}_{3}\right) \delta: 7.99(\mathrm{~d}, J=2.0 \mathrm{~Hz}, 1 \mathrm{H})$, $7.73(\mathrm{dd}, J=8.5,2.0 \mathrm{~Hz}, 1 \mathrm{H}), 6.95(\mathrm{~d}, J=8.5 \mathrm{~Hz}, 1 \mathrm{H})$, $6.94 \sim 6.90(\mathrm{~m}, 2 \mathrm{H}), 6.87 \sim 6.83(\mathrm{~m}, 2 \mathrm{H}), 6.56(\mathrm{~s}, 1 \mathrm{H})$, $5.13(\mathrm{~s}, 2 \mathrm{H}), 3.94(\mathrm{~s}, 3 \mathrm{H}), 3.77(\mathrm{~s}, 3 \mathrm{H}) ;{ }^{13} \mathrm{C}$ NMR $(126$ $\left.\mathrm{MHz}, \mathrm{CDCl}_{3}\right) \delta: 168.95,161.01,157.25,154.69,151.89$, $131.76,127.18,122.62,116.04,114.80,112.17,111.96$, $101.00,62.29,56.36,55.70$.

3-(3-甲氧基苯基)-4-溴-5-(3-硝基苯氧甲基)异噁唑 (3-8): 白色固体, 产率 82.8\%. m.p. $171.4 \sim 173.7{ }^{\circ} \mathrm{C} ;{ }^{1} \mathrm{H}$ NMR (500 MHz, $\left.\mathrm{CDCl}_{3}\right) \delta: 8.00(\mathrm{~d}, J=2.0 \mathrm{~Hz}, 1 \mathrm{H}), 7.91$ $(\mathrm{d}, J=8.0,1 \mathrm{H}), 7.85(\mathrm{t}, J=2.0 \mathrm{~Hz}, 1 \mathrm{H}), 7.75(\mathrm{dd}, J=8.5$, $2.0 \mathrm{~Hz}, 1 \mathrm{H}), 7.49$ (t, $J=8.0 \mathrm{~Hz}, 1 \mathrm{H}), 7.32(\mathrm{dd}, J=8.5,2.0$ $\mathrm{Hz}, 1 \mathrm{H}), 6.97$ (d, $J=8.5 \mathrm{~Hz}, 1 \mathrm{H}), 6.64(\mathrm{~s}, 1 \mathrm{H}), 5.28(\mathrm{~s}$, $2 \mathrm{H}), 3.95(\mathrm{~s}, 3 \mathrm{H}) ;{ }^{13} \mathrm{C} \mathrm{NMR}\left(126 \mathrm{MHz}, \mathrm{CDCl}_{3}\right) \delta: 165.22$, $159.49,154.80,151.89,148.49,135.00,129.80,129.40$, $129.15,128.84,128.26,123.80,119.36,116.40,114.75$, $60.62,55.71$.

3-(3-甲氧基苯基)-4-溴-5-(4-氟苯氧甲基)异噁唑 (3-9): 白色固体, 产率 88.5\%. m.p. $115.5 \sim 116.0{ }^{\circ} \mathrm{C} ;{ }^{1} \mathrm{H}$ NMR (500 MHz, $\left.\mathrm{CDCl}_{3}\right) \delta: 7.99(\mathrm{~d}, J=2.0 \mathrm{~Hz}, 1 \mathrm{H}), 7.74$ $(\mathrm{dd}, J=8.5,2.0 \mathrm{~Hz}, 1 \mathrm{H}), 7.04 \sim 6.98(\mathrm{~m}, 2 \mathrm{H}), 6.96(\mathrm{~d}, J=$ $8.5 \mathrm{~Hz}, 1 \mathrm{H}), 6.95 \sim 6.91(\mathrm{~m}, 2 \mathrm{H}), 6.58(\mathrm{~s}, 1 \mathrm{H}), 5.16(\mathrm{~d}, J=$ $0.5 \mathrm{~Hz}, 2 \mathrm{H}), 3.95$ (s, 3H). ${ }^{19} \mathrm{~F}$ NMR (471 MHz, $\left.\mathrm{CDCl}_{3}\right) \delta$ : $-122.11 \sim-122.27(\mathrm{~m}) ;{ }^{13} \mathrm{C}$ NMR (126 MHz, DMSO- $\left.d_{6}\right)$ $\delta: 168.87,161.01,157.48\left(\mathrm{~d},{ }^{1} J_{\mathrm{C}-\mathrm{F}}=237.5 \mathrm{~Hz}, \mathrm{ArF}\right)$, $157.31,154.32,131.37,127.95,122.51,116.70\left(\mathrm{~d},{ }^{3} J_{\mathrm{C}-\mathrm{F}}=\right.$ $8.1 \mathrm{~Hz}, \mathrm{ArF}), 116.42\left(\mathrm{~d},{ }^{2} J_{\mathrm{C}-\mathrm{F}}=23.3 \mathrm{~Hz}, \mathrm{ArF}\right), 113.40$, $111.76,102.63,61.54,56.86$.

3-(3-甲氧基苯基)-4-溴-5-(3-甲基苯氧甲基)异啞唑 (3-10): 白色固体, 产率 84.9\%. m.p. $107.7 \sim 108.3{ }^{\circ} \mathrm{C}$; ${ }^{1} \mathrm{H}$ NMR $\left(500 \mathrm{MHz}, \mathrm{CDCl}_{3}\right) \delta: 8.00(\mathrm{~d}, J=2.0 \mathrm{~Hz}, 1 \mathrm{H})$, $7.74(\mathrm{dd}, J=8.5,2.0 \mathrm{~Hz}, 1 \mathrm{H}), 7.20(\mathrm{t}, J=8.0 \mathrm{~Hz}, 1 \mathrm{H}), 6.96$ $(\mathrm{d}, J=8.5 \mathrm{~Hz}, 1 \mathrm{H}), 6.85 \sim 6.77(\mathrm{~m}, 3 \mathrm{H}), 6.58(\mathrm{~s}, 1 \mathrm{H}), 5.18$ (s, 2H), $3.95(\mathrm{~s}, 3 \mathrm{H}), 2.35(\mathrm{~s}, 3 \mathrm{H}) ;{ }^{13} \mathrm{C} \mathrm{NMR}(126 \mathrm{MHz}$, $\left.\mathrm{CDCl}_{3}\right) \delta: 168.85,161.02,157.82,157.24,139.87,131.73$, $129.45,127.21,122.76,122.60,115.66,112.17,111.97$, $111.56,100.99,61.34,56.35,21.56$.

\section{2 .3 化合物 $\mathbf{5}$ 的合成}

称取 3-氰基苯基-4-溴-5-(3-硝基苯氧甲基)异噁唑 $(0.4 \mathrm{~g}, 1.00 \mathrm{mmol}) 、$ 苯喼酸 $(0.18 \mathrm{~g}, 1.50 \mathrm{mmol}) 、 \mathrm{~K}_{2} \mathrm{CO}_{3}$ $(0.20 \mathrm{~g}, 2.00 \mathrm{mmol}) 、 \mathrm{Pd}\left(\mathrm{PPh}_{3}\right)_{2} \mathrm{Cl}_{2}(0.07 \mathrm{~g}, 0.10 \mathrm{mmol})$ 于 $25 \mathrm{~mL}$ 圆底烧瓶中, 加入 $10 \mathrm{~mL}$ 甲苯和 $1 \mathrm{~mL}$ 水, 在氮气 保护下, $90{ }^{\circ} \mathrm{C}$ 反应 $12 \mathrm{~h}$, 停止反应冷却至室温. 将反应 液倒入水中并用乙酸乙酯萃取 $(30 \mathrm{~mL} \times 3)$, 收集有机相, 用无水 $\mathrm{MgSO}_{4}$ 干燥后浓缩得粗产物, 粗产物用 $V($ 乙酸 乙酯) : $V$ (石油 醚 $)=1: 8$ 为淋洗剂, 经柱层析分离得 4-氧基苯基-4-苯基-5-(3-硝基苯氧甲基)异啞唑(5-1) 344 $\mathrm{mg}$, 产率为 $88 \%$. 化合物 5-2 5-30 按上述方法合成.

3-(4-氰基苯基)-4-苯基-5-(4-硝基苯氧甲基)异啞唑 (5-1): 白色固体, 产率 88\%. m.p. 155.9 $157.0{ }^{\circ} \mathrm{C} ;{ }^{1} \mathrm{H}$ NMR (500 MHz, $\left.\mathrm{CDCl}_{3}\right) \delta: 8.21(\mathrm{dd}, J=7.0,2.0 \mathrm{~Hz}, 2 \mathrm{H})$, $7.63(\mathrm{~d}, J=8.0 \mathrm{~Hz}, 2 \mathrm{H}), 7.58$ (d, $J=8.0 \mathrm{~Hz}, 2 \mathrm{H}), 7.45 \sim$ $7.41(\mathrm{~m}, 3 \mathrm{H}), 7.21(\mathrm{dd}, J=7.0,2.0 \mathrm{~Hz}, 2 \mathrm{H}), 7.00(\mathrm{dd}, J=$ 7.5, $2.0 \mathrm{~Hz}, 2 \mathrm{H}), 5.21(\mathrm{~s}, 2 \mathrm{H}) ;{ }^{13} \mathrm{C} \mathrm{NMR}(126 \mathrm{MHz}$, $\left.\mathrm{CDCl}_{3}\right) \delta: 163.40,162.42,159.91,142.38,132.77,132.43$, 
$129.68,129.33,129.15,129.04,127.92,125.99,119.97$, 118.20, 114.84, 113.66, 59.87; IR (KBr) v: 3078, 2930, 2836, 2224, 1592, 1502, 1334, 1249, 1107, 1012, 845 $\mathrm{cm}^{-1}$. HRMS (ESI) calcd for $\mathrm{C}_{23} \mathrm{H}_{16} \mathrm{~N}_{3} \mathrm{O}_{4}\left[(\mathrm{M}+\mathrm{H})^{+}\right]$: 398.1133, found 398.1133.

3-(4-氧基苯基)-4-(4-氟苯基)-5-(4-硝基苯氧甲基)异 噁唑(5-2): 白色固体, 产率 $86 \%$. m.p. $164.0 \sim 165.3{ }^{\circ} \mathrm{C}$; ${ }^{1} \mathrm{H}$ NMR $\left(500 \mathrm{MHz}, \mathrm{CDCl}_{3}\right) \delta: 8.22(\mathrm{dd}, J=7.0,2.0 \mathrm{~Hz}$, 2H), 7.65 (d, $J=9.0 \mathrm{~Hz}, 2 \mathrm{H}), 7.57$ (d, $J=8.5 \mathrm{~Hz}, 2 \mathrm{H}), 7.21$ (dd, $J=9.0,5.0 \mathrm{~Hz}, 2 \mathrm{H}), 7.13$ (t, $J=8.5 \mathrm{~Hz}, 2 \mathrm{H}), 7.02$ (d, $J=8.5 \mathrm{~Hz}, 2 \mathrm{H}), 5.19$ (s, 2H); ${ }^{19} \mathrm{~F} \mathrm{NMR}\left(471 \mathrm{MHz}, \mathrm{CDCl}_{3}\right)$ $\delta:-111.82 .{ }^{13} \mathrm{C}$ NMR $\left(126 \mathrm{MHz}, \mathrm{CDCl}_{3}\right) \delta: 163.55$, $163.06\left(\mathrm{~d},{ }^{1} J_{\mathrm{C}-\mathrm{F}}=250.7 \mathrm{~Hz}, \mathrm{ArF}\right), 162.07,159.91,142.40$, $132.61,132.51,131.57\left(\mathrm{~d},{ }^{3} J_{\mathrm{C}-\mathrm{F}}=8.3 \mathrm{~Hz}, \mathrm{ArF}\right), 129.01$, $126.01,123.89,123.86,119.01,118.14,116.55\left(\mathrm{~d},{ }^{2} J_{\mathrm{C}-\mathrm{F}}=\right.$ $21.7 \mathrm{~Hz}, \mathrm{ArF}), 114.83,113.75,59.89$; IR (KBr) v: 3084, 2925, 2850, 2228, 1596, 1509, 1340, 1257, 1105, 842 $\mathrm{cm}^{-1}$. HRMS (ESI) calcd for $\mathrm{C}_{23} \mathrm{H}_{15} \mathrm{FN}_{3} \mathrm{O}_{4}\left[(\mathrm{M}+\mathrm{H})^{+}\right]$: 416.1044, found 416.1041.

3-(4-氧基苯基)-4-(4-三氟甲基苯基)-5-(3-硝基苯氧 甲基)异噁唑(5-3): 白色固体, 产率 91\%. m.p. 131.0 $131.3{ }^{\circ} \mathrm{C} ;{ }^{1} \mathrm{H}$ NMR $\left(500 \mathrm{MHz}, \mathrm{CDCl}_{3}\right) \delta$ : 7.94 (ddd, $J=$ 8.0, 2.0, $1.0 \mathrm{~Hz}, 1 \mathrm{H}), 7.79$ (t, $J=2.0 \mathrm{~Hz}, 1 \mathrm{H}), 7.75 \sim 7.68$ (m, 4H), 7.59 (dd, $J=8.0,2.0 \mathrm{~Hz}, 2 \mathrm{H}), 7.51(\mathrm{t}, J=8.0 \mathrm{~Hz}$, 1H), 7.40 (d, $J=8.0 \mathrm{~Hz}, 2 \mathrm{H}$ ), 7.33 (ddd, $J=8.0,2.0,1.0$ $\mathrm{Hz}, 1 \mathrm{H}), 5.22(\mathrm{~s}, 2 \mathrm{H}) ;{ }^{19} \mathrm{~F}$ NMR (471 $\left.\mathrm{MHz}, \mathrm{CDCl}_{3}\right) \delta$ : $-62.81 ;{ }^{13} \mathrm{C}$ NMR $\left(126 \mathrm{MHz}, \mathrm{CDCl}_{3}\right) \delta: 164.30,159.87$, $158.01,149.15,132.60,132.37,131.88,131.12\left(\mathrm{q},{ }^{2} J_{\mathrm{C}-\mathrm{F}}=\right.$ $\left.32.9 \mathrm{~Hz}, \mathrm{ArCF}_{3}\right), 130.43,130.17,129.09,126.24$ (q, $\left.{ }^{3} J_{\mathrm{C}^{-\mathrm{F}}}=3.6 \mathrm{~Hz}, \mathrm{ArCF}_{3}\right), 123.74\left(\mathrm{q},{ }^{1} J_{\mathrm{C}-\mathrm{F}}=273.4 \mathrm{~Hz}, \mathrm{CF}_{3}\right)$, 121.86, 118.61, 118.08, 117.09, 113.88, 109.16, 60.00; IR (KBr) $v$ : 3101, 2925, 2855, 2228, 1622, 1523, 1331, 1241, $1115,844 \mathrm{~cm}^{-1}$. HRMS (ESI) calcd for $\mathrm{C}_{24} \mathrm{H}_{15} \mathrm{~F}_{3} \mathrm{~N}_{3} \mathrm{O}_{4}$ $\left[(\mathrm{M}+\mathrm{H})^{+}\right]:$466.1004, found 466.1009 .

3-(4-氧基苯基)-4-苯基-5-(4-甲氧基苯氧甲基)异噁 唑(5-4): 白色固体, 产率 82\%. m.p. 104.1 105.1 ${ }^{\circ} \mathrm{C} ;{ }^{1} \mathrm{H}$ NMR $\left(500 \mathrm{MHz}, \mathrm{CDCl}_{3}\right) \delta: 7.64 \sim 7.60(\mathrm{~m}, 4 \mathrm{H}), 7.43 \sim$ $7.37(\mathrm{~m}, 3 \mathrm{H}), 7.20(\mathrm{dt}, J=6.0,2.0 \mathrm{~Hz}, 2 \mathrm{H}), 6.88$ (dd, $J=$ 6.0, $2.0 \mathrm{~Hz}, 2 \mathrm{H}), 6.82(\mathrm{dd}, J=6.0,2.0 \mathrm{~Hz}, 2 \mathrm{H}), 5.04(\mathrm{~s}$, $2 \mathrm{H}), 3.77(\mathrm{~s}, 3 \mathrm{H}) ;{ }^{13} \mathrm{C} \mathrm{NMR}\left(126 \mathrm{MHz}, \mathrm{CDCl}_{3}\right) \delta: 165.11$, $159.73,154.78,151.89,133.20,132.37,129.79,129.11$, $129.08,128.77,128.34,119.26,118.32,116.39,114.74$, 113.41, 60.62, 55.71; IR (KBr) v: 3051, 2932, 2228, 1640, $1505,1448,1331,1228,1028,837 \mathrm{~cm}^{-1}$. HRMS (ESI) calcd for $\mathrm{C}_{24} \mathrm{H}_{19} \mathrm{~N}_{2} \mathrm{O}_{3}\left[(\mathrm{M}+\mathrm{H})^{+}\right]$: 383.1351, found
383.1354 .

3-(4-氰基苯基)-4-(4-氟苯基)-5-(4-甲氧基苯氧甲基) 异噁唑(5-5): 白色固体, 产率 91\%. m.p. 85.1 85.7 ${ }^{\circ} \mathrm{C}$; ${ }^{1} \mathrm{H}$ NMR $\left(500 \mathrm{MHz}, \mathrm{CDCl}_{3}\right) \delta: 7.65(\mathrm{~d}, J=2.0 \mathrm{~Hz}, 2 \mathrm{H})$, 7.57 (dd, $J=6.5,2.0 \mathrm{~Hz}, 2 \mathrm{H}), 7.21 \sim 7.15(\mathrm{~m}, 2 \mathrm{H}), 7.09$ (dd, $J=6.5,2.0 \mathrm{~Hz}, 2 \mathrm{H}), 6.88(\mathrm{dd}, J=6.5,2.5 \mathrm{~Hz}, 2 \mathrm{H})$, 6.86 (dd, $J=6.5,2.5 \mathrm{~Hz}, 2 \mathrm{H}), 5.03$ (s, 2H), 3.77 (s, 3H); ${ }^{19} \mathrm{~F}$ NMR $\left(471 \mathrm{MHz}, \mathrm{CDCl}_{3}\right) \delta:-111.97 \sim-111.04(\mathrm{~m})$; ${ }^{13} \mathrm{C} \mathrm{NMR}\left(126 \mathrm{MHz}, \mathrm{CDCl}_{3}\right) \delta: 165.24,162.89\left(\mathrm{~d},{ }^{1} J_{\mathrm{C}-\mathrm{F}}=\right.$ $249.8 \mathrm{~Hz}, \mathrm{ArF}), 159.72,154.84,151.79,133.00,132.45$, $131.65\left(\mathrm{~d},{ }^{3} J_{\mathrm{C}-\mathrm{F}}=8.7 \mathrm{~Hz}, \mathrm{ArF}\right), 129.04,124.32,118.31$, $118.24,116.38,116.27\left(\mathrm{~d},{ }^{2} J_{\mathrm{C}-\mathrm{F}}=21.9 \mathrm{~Hz}, \mathrm{ArF}\right), 114.77$, 113.53, 60.64, 55.69; IR (KBr) v: 3062, 2942, 2826, 2225 , $1507,1331,1230,1050,829 \mathrm{~cm}^{-1}$. HRMS (ESI) calcd for $\mathrm{C}_{24} \mathrm{H}_{18} \mathrm{FN}_{2} \mathrm{O}_{3}\left[(\mathrm{M}+\mathrm{H})^{+}\right]: 401.1257$, found 401.1259 .

3-(4-氰基苯基)-4-(4-三氟甲基苯基)-5-(4-甲氧基苯 氧甲)异噁唑(5-6): 白色固体, 产率 92\%. m.p. 104.2 $105.1{ }^{\circ} \mathrm{C} ;{ }^{1} \mathrm{H}$ NMR $\left(500 \mathrm{MHz}, \mathrm{CDCl}_{3}\right) \delta: 7.68 \sim 7.63(\mathrm{~m}$, 4H), $7.55(\mathrm{dd}, J=6.5,2.5 \mathrm{~Hz}, 2 \mathrm{H}), 7.34(\mathrm{~d}, J=8.0 \mathrm{~Hz}$, $2 \mathrm{H}), 6.87 \sim 6.90(\mathrm{~m}, 4 \mathrm{H}), 5.05(\mathrm{~s}, 2 \mathrm{H}), 3.77(\mathrm{~s}, 3 \mathrm{H}) ;{ }^{19} \mathrm{~F}$ NMR $\left(471 \mathrm{MHz}, \mathrm{CDCl}_{3}\right) \delta:-62.75 ;{ }^{13} \mathrm{C}$ NMR $(126$ $\left.\mathrm{MHz}, \mathrm{CDCl}_{3}\right) \delta: 165.75,159.73,154.93,151.67,132.67$, 132.56, 132.24, 130.85 (q, ${ }^{2} J_{\mathrm{C}-\mathrm{F}}=32.7 \mathrm{~Hz}, \mathrm{ArCF}_{3}$ ), 130.15, 129.11, 126.03(q, ${ }^{3} J_{\mathrm{C}-\mathrm{F}}=3.6 \mathrm{~Hz}, \mathrm{ArCF}_{3}$ ), 123.81 (q, $\left.{ }^{1} J_{\mathrm{C}-\mathrm{F}}=273.4 \mathrm{~Hz}, \mathrm{CF}_{3}\right), 118.13,118.03,116.36,114.80$, 113.76, 60.69, 55.68; IR (KBr) v: 3062, 2938, 2842, 2229, $1506,1324,1231,1127,839 \mathrm{~cm}^{-1}$. HRMS (ESI) calcd for $\mathrm{C}_{25} \mathrm{H}_{18} \mathrm{~F}_{3} \mathrm{~N}_{2} \mathrm{O}_{3}\left[(\mathrm{M}+\mathrm{H})^{+}\right]$: 451.1225, found 451.1229.

3-(4-氧基苯基)-4-苯基-5-(4-氟苯氧甲基)异噁唑 (5-7): 白色固体, 产率 $89 \%$. m.p. $103.8 \sim 104.5{ }^{\circ} \mathrm{C} ;{ }^{1} \mathrm{H}$ NMR (500 MHz, $\left.\mathrm{CDCl}_{3}\right) \delta: 7.64(\mathrm{~d}, J=7.5 \mathrm{~Hz}, 2 \mathrm{H}), 7.58$ (d, $J=7.5 \mathrm{~Hz}, 2 \mathrm{H}), 7.43 \sim 7.38$ (m, 3H), 7.20 (ddd, $J=7.0$, $2.0,1.0 \mathrm{~Hz}, 2 \mathrm{H}), 7.00(\mathrm{dt}, J=7.0,2.0 \mathrm{~Hz}, 2 \mathrm{H}), 6.91 \sim 6.83$ (m, 2H), $5.06(\mathrm{~s}, 2 \mathrm{H}) ;{ }^{19} \mathrm{~F}$ NMR (471 MHz, $\left.\mathrm{CDCl}_{3}\right) \delta$ : -122.02 (s); ${ }^{13} \mathrm{C}$ NMR (126 MHz, $\left.\mathrm{CDCl}_{3}\right) \delta: 164.67$, 159.78, $157.95\left(\mathrm{~d},{ }^{1} J_{\mathrm{C}-\mathrm{F}}=240.52 \mathrm{~Hz}, \mathrm{ArF}\right), 153.87$, $133.08,132.39$, 129.76, 129.17, 129.07, 128.88, 128.23, $119.43,118.29,116.35\left(\mathrm{~d},{ }^{3} J_{\mathrm{C}-\mathrm{F}}=8.19 \mathrm{~Hz}, \operatorname{ArF}\right), 116.06(\mathrm{~d}$, $\left.{ }^{2} J_{\mathrm{C}-\mathrm{F}}=23.31 \mathrm{~Hz}, \mathrm{ArF}\right), 113.48,60.42$; IR $(\mathrm{KBr}) v: 3070$, 2917, 2864, 2225, 1500, 1331, 1203, 1032, $825 \mathrm{~cm}^{-1}$. HRMS (ESI) calcd for $\mathrm{C}_{23} \mathrm{H}_{16} \mathrm{FN}_{2} \mathrm{O}_{2}\left[(\mathrm{M}+\mathrm{H})^{+}\right]$: 371.1194 , found 371.1190 .

3-(4-氧基苯基)-4-(4-氟苯基)-5-(4-氟苯氧甲基)异噁 唑(5-8): 白色固体, 产率 $82 \%$ m.p. $113.7 \sim 114.1{ }^{\circ} \mathrm{C} ;{ }^{1} \mathrm{H}$ NMR (500 MHz, $\left.\mathrm{CDCl}_{3}\right) \delta: 7.64(\mathrm{~d}, J=8.0 \mathrm{~Hz}, 2 \mathrm{H}), 7.57$ 
(d, $J=8.0 \mathrm{~Hz}, 2 \mathrm{H}), 7.23 \sim 7.15(\mathrm{~m}, 2 \mathrm{H}), 7.10(\mathrm{~m}, 2 \mathrm{H})$, $7.02 \sim 6.95(\mathrm{~m}, 2 \mathrm{H}), 6.92 \sim 6.84(\mathrm{~m}, 2 \mathrm{H}), 5.05(\mathrm{~s}, 2 \mathrm{H}) ;{ }^{19} \mathrm{~F}$ NMR $\left(471 \mathrm{MHz}, \mathrm{CDCl}_{3}\right) \delta:-111.76 \sim-111.79(\mathrm{~m})$, $-121.85 \sim-121.89(\mathrm{~m}) ;{ }^{13} \mathrm{C}$ NMR $\left(126 \mathrm{MHz}, \mathrm{CDCl}_{3}\right) \delta$ : $164.79,162.93\left(\mathrm{~d},{ }^{1} J_{\mathrm{C}-\mathrm{F}}=249.7 \mathrm{~Hz}, \mathrm{ArF}\right), 159.78,157.95$ $\left(\mathrm{d},{ }^{1} J_{\mathrm{C}-\mathrm{F}}=240.4 \mathrm{~Hz}, \mathrm{ArF}\right), 153.80,132.88,132.46,131.63$ $\left(\mathrm{d},{ }^{3} J_{\mathrm{C}-\mathrm{F}}=8.3 \mathrm{~Hz}, \mathrm{ArF}\right), 129.03,124.21,118.47,118.22$, $116.34\left(\mathrm{~d},{ }^{2} J_{\mathrm{C}-\mathrm{F}}=21.8 \mathrm{~Hz}, \mathrm{ArF}\right), 116.30,116.27\left(\mathrm{~d},{ }^{2} J_{\mathrm{C}^{-\mathrm{F}}}=\right.$ $21.7 \mathrm{~Hz}, \mathrm{ArF}), 116.01,113.58,60.42$; IR (KBr) v: 3076, 2923, 2857, 2227, 1601, 1501, 1205, 1115, 834 $\mathrm{cm}^{-1}$. HRMS (ESI) calcd for $\mathrm{C}_{23} \mathrm{H}_{15} \mathrm{~F}_{2} \mathrm{~N}_{2} \mathrm{O}_{2}\left[(\mathrm{M}+\mathrm{H})^{+}\right]$: 389.1100 , found 389.1102 .

3-(4-氰基苯基)-4-(4-三氟甲基苯基) -5-(4-氟苯氧甲 基)异噁唑(5-9): 白色固体, 产率 85\%. m.p. 114.6 $115.1{ }^{\circ} \mathrm{C} ;{ }^{1} \mathrm{H}$ NMR $\left(500 \mathrm{MHz}, \mathrm{CDCl}_{3}\right) \delta: 7.70 \sim 7.61(\mathrm{~m}$, $4 \mathrm{H}), 7.59 \sim 7.51(\mathrm{~m}, 2 \mathrm{H}), 7.34(\mathrm{~d}, J=2.0 \mathrm{~Hz}, 2 \mathrm{H}), 6.96(\mathrm{t}$, $J=8.0 \mathrm{~Hz}, 2 \mathrm{H}), 6.90 \sim 6.85(\mathrm{~m}, 2 \mathrm{H}), 5.07(\mathrm{~s}, 2 \mathrm{H}) ;{ }^{19} \mathrm{~F}$ NMR $\left(471 \mathrm{MHz}, \mathrm{CDCl}_{3}\right) \delta$ : $-62.77(\mathrm{~s}),-121.63 \sim$ $121.69(\mathrm{~m}) ;{ }^{13} \mathrm{C}$ NMR $\left(126 \mathrm{MHz}, \mathrm{CDCl}_{3}\right) \delta: 165.29$, $159.79,158.04\left(\mathrm{~d},{ }^{1} J_{\mathrm{C}-\mathrm{F}}=240.2 \mathrm{~Hz}, \mathrm{ArF}\right), 153.69,132.58$, $132.14,130.95$ (q, ${ }^{2} J=32.7 \mathrm{~Hz}, \mathrm{ArCF}_{3}$ ), 130.14, 129.10 , $126.10\left(\mathrm{q},{ }^{3} J=3.6 \mathrm{~Hz}, \mathrm{ArCF}_{3}\right), 123.79$, (q, ${ }^{1} J_{\mathrm{C}-\mathrm{F}}=273.4$ $\left.\mathrm{Hz}, \mathrm{CF}_{3}\right), 118.19,118.11,116.30\left(\mathrm{~d},{ }^{3} J_{\mathrm{C}^{-} \mathrm{F}}=8.2 \mathrm{~Hz}, \mathrm{ArF}\right)$, $116.16\left(\mathrm{~d},{ }^{2} J_{\mathrm{C}-\mathrm{F}}=23.3 \mathrm{~Hz}, \mathrm{ArF}\right), 113.82,60.46$; IR (KBr) v: 3076, 2923, 2857, 2227, 1601, 1501, 1205, 1115, 834 $\mathrm{cm}^{-1}$. HRMS (ESI) calcd for $\mathrm{C}_{24} \mathrm{H}_{15} \mathrm{~F}_{4} \mathrm{~N}_{2} \mathrm{O}_{2}\left[(\mathrm{M}+\mathrm{H})^{+}\right]$: 439.1062, found 439.1064.

3-(4-硝基)-4-苯基-5-(4-甲氧基苯氧甲基)异噁唑 (5-10): 白色固体, 产率 $85 \%$. m.p. 121.6 122.3 ${ }^{\circ} \mathrm{C} ;{ }^{1} \mathrm{H}$ NMR $\left(500 \mathrm{MHz}, \mathrm{CDCl}_{3}\right) \delta: 8.19(\mathrm{~d}, J=9.0 \mathrm{~Hz}, 2 \mathrm{H}), 7.68$ (d, $J=9.0 \mathrm{~Hz}, 2 \mathrm{H}), 7.43 \sim 7.39$ (m, 3H), 7.21 (dd, $J=8.0$, $2.0 \mathrm{~Hz}, 2 \mathrm{H}), 6.91(\mathrm{dd}, J=4.0,2.0 \mathrm{~Hz}, 2 \mathrm{H}), 6.86 \sim 6.83(\mathrm{~m}$, $2 \mathrm{H}), 5.05(\mathrm{~s}, 2 \mathrm{H}), 3.77(\mathrm{~s}, 3 \mathrm{H}) ;{ }^{13} \mathrm{C}$ NMR (126 MHz, $\left.\mathrm{CDCl}_{3}\right) \delta: 165.23,159.49,154.78,151.88,148.48,135.00$, $129.81,129.41,129.16,128.84,128.27,123.80,119.36$, 116.38, 114.74, 60.61, 55.70; IR (KBr) v: 3063, 2933, 2848, 1603, 1511, 1350, 1226, 1027, $846 \mathrm{~cm}^{-1}$. HRMS (ESI) calcd for $\mathrm{C}_{23} \mathrm{H}_{19} \mathrm{~N}_{2} \mathrm{O}_{5}\left[(\mathrm{M}+\mathrm{H})^{+}\right]$: 403.1249 , found 403.1251 .

3-(4-硝基苯基)-4-(4-氟苯基)-5-(4-甲氧基苯氧甲基) 异噁唑 (5-11): 白色固体, 产率 83\%. m.p. 121.8 $122.6{ }^{\circ} \mathrm{C} ;{ }^{1} \mathrm{H}$ NMR $\left(500 \mathrm{MHz}, \mathrm{CDCl}_{3}\right) \delta: 8.20(\mathrm{~d}, J=9.0$ $\mathrm{Hz}, 2 \mathrm{H}), 7.63(\mathrm{~d}, J=9.0 \mathrm{~Hz}, 2 \mathrm{H}), 7.08(\mathrm{dd}, J=9.0,5.0 \mathrm{~Hz}$, $2 \mathrm{H}), 7.10(\mathrm{t}, J=9.0 \mathrm{~Hz}, 2 \mathrm{H}), 6.82 \sim 6.81(\mathrm{~m}, 4 \mathrm{H}), 5.04(\mathrm{~s}$, $2 \mathrm{H}), 3.77(\mathrm{~s}, 3 \mathrm{H}) ;{ }^{19} \mathrm{~F}$ NMR (471 $\left.\mathrm{MHz}, \mathrm{CDCl}_{3}\right) \delta$ :
-111.89 (s); ${ }^{13} \mathrm{C}$ NMR (126 MHz, $\left.\mathrm{CDCl}_{3}\right) \delta: 165.34$, $162.93\left(\mathrm{~d},{ }^{1} J_{\mathrm{C}-\mathrm{F}}=249.8 \mathrm{~Hz}, \mathrm{ArF}\right), 159.48,154.86,151.78$, $148.55,134.79,131.64\left(\mathrm{~d},{ }^{3} J_{\mathrm{C}-\mathrm{F}}=8.25 \mathrm{~Hz}, \mathrm{ArF}\right), 129.37$, $124.22,123.88,118.41,116.33\left(\mathrm{~d},{ }^{2} J_{\mathrm{C}-\mathrm{F}}=21.86 \mathrm{~Hz}, \mathrm{ArF}\right)$, 116.38, 114.78, 60.63, 55.71; IR (KBr) v: 3078, 2945, 2834, 1595, 1509, 1350,1216, 1031, $846 \mathrm{~cm}^{-1}$. HRMS (ESI) calcd for $\mathrm{C}_{23} \mathrm{H}_{18} \mathrm{FN}_{2} \mathrm{O}_{5}\left[(\mathrm{M}+\mathrm{H})^{+}\right]$: 421.1191, found 421.1194 .

3-(4-硝基苯基)-4-(4-三氟甲基苯基)-5-(4-甲氧基苯 氧甲)异噁唑(5-12): 白色固体, 产率 86\%. m.p. 120.8 $121.7{ }^{\circ} \mathrm{C} ;{ }^{1} \mathrm{H}$ NMR $\left(500 \mathrm{MHz}, \mathrm{CDCl}_{3}\right) \delta: 8.20(\mathrm{~d}, J=9.0$ $\mathrm{Hz}, 2 \mathrm{H}), 7.63(\mathrm{~d}, J=8.5 \mathrm{~Hz}, 2 \mathrm{H}), 7.20(\mathrm{dd}, J=9.0,5.0 \mathrm{~Hz}$, $2 \mathrm{H}), 7.10(\mathrm{t}, J=8.5 \mathrm{~Hz}, 2 \mathrm{H}), 6.87(\mathrm{~d}, J=9.5 \mathrm{~Hz}, 2 \mathrm{H}), 6.83$ (d, $J=9.5 \mathrm{~Hz}, 2 \mathrm{H}), 5.04$ (s, 2H), 3.77 (s, 3H); ${ }^{19} \mathrm{~F}$ NMR $\left(471 \mathrm{MHz}, \mathrm{CDCl}_{3}\right) \delta:-62.76(\mathrm{~s}) ;{ }^{13} \mathrm{C}$ NMR $(126 \mathrm{MHz}$, $\left.\mathrm{CDCl}_{3}\right) \delta: 165.84,159.48,154.95,151.65,148.67,134.45$, $132.14,130.96\left(\mathrm{q},{ }^{2} J_{\mathrm{C}^{-} \mathrm{F}}=33.0 \mathrm{~Hz}\right), 130.15,129.45,126.08$ $\left(\mathrm{q},{ }^{3} J_{\mathrm{C}-\mathrm{F}}=3.7 \mathrm{~Hz}\right), 124.00,123.78\left(\mathrm{q},{ }^{1} J_{\mathrm{C}-\mathrm{F}}=270.9 \mathrm{~Hz}\right)$, 118.13, 116.35, 114.81, 60.67, 55.70; IR (KBr) v: 3074, 2915, 2843, 1607, 1513, 1333, 1230, 1116, $850 \mathrm{~cm}^{-1}$. HRMS (ESI) calcd for $\mathrm{C}_{24} \mathrm{H}_{18} \mathrm{~F}_{3} \mathrm{~N}_{2} \mathrm{O}_{5}\left[(\mathrm{M}+\mathrm{H})^{+}\right]$: 471.1123, found 471.1125 .

3-(4-硝基苯基)-4-苯基-5-(3-硝基苯氧甲基)异噁坐 (5-13): 白色固体, 产率 84\%. m.p. $137.9 \sim 138.8{ }^{\circ} \mathrm{C} ;{ }^{1} \mathrm{H}$ NMR $\left(500 \mathrm{MHz}, \mathrm{CDCl}_{3}\right) \delta: 8.18(\mathrm{~d}, J=9.0 \mathrm{~Hz}, 2 \mathrm{H}), 7.88$ (dd, $J=8.0,1.0 \mathrm{~Hz}, 1 \mathrm{H}), 7.75(\mathrm{~d}, J=2.5 \mathrm{~Hz}, 1 \mathrm{H}), 7.66(\mathrm{~d}$, $J=8.5 \mathrm{~Hz}, 2 \mathrm{H}), 7.47 \sim 7.41$ (m, 4H), 7.28 (dd, $J=8.0,2.0$ $\mathrm{Hz}, 1 \mathrm{H}), 7.24 \sim 7.22(\mathrm{~m}, 2 \mathrm{H}), 5.21(\mathrm{~s}, 2 \mathrm{H}) ;{ }^{13} \mathrm{C}$ NMR $(126$ $\left.\mathrm{MHz}, \mathrm{CDCl}_{3}\right) \delta: 163.80,159.61,158.16,149.15,148.54$, $134.68,130.32$, 129.75, 129.39, 129.35, 129.15, 127.86, 123.84, 121.91, 119.95, 116.94, 109.31, 60.01; IR (KBr) v: 3074, 2915, 2843, 1607, 1513, 1333, 1230, 1116, 850 $\mathrm{cm}^{-1}$. HRMS (ESI) calcd for $\mathrm{C}_{22} \mathrm{H}_{16} \mathrm{~N}_{3} \mathrm{O}_{6}\left[(\mathrm{M}+\mathrm{H})^{+}\right]$: 418.1005, found 418.1009.

3-(4-硝基苯基)-4-(4-氟苯基)-5-(3-硝基苯氧甲基)异 啞坐(5-14): 白色固体, 产率 $81 \%$. m.p. $130.4 \sim 131.3{ }^{\circ} \mathrm{C}$; ${ }^{1} \mathrm{H}$ NMR $\left(500 \mathrm{MHz}, \mathrm{CDCl}_{3}\right) \delta: 8.22 \sim 8.20(\mathrm{~m}, 2 \mathrm{H})$, $7.90 \sim 7.88(\mathrm{~m}, 1 \mathrm{H}), 7.76(\mathrm{t}, J=2.5 \mathrm{~Hz}, 1 \mathrm{H}), 7.65 \sim 7.63$ $(\mathrm{m}, 2 \mathrm{H}), 7.48(\mathrm{t}, J=8.0 \mathrm{~Hz}, 1 \mathrm{H}), 7.30 \sim 7.28(\mathrm{~m}, 1 \mathrm{H})$, $7.24 \sim 7.21(\mathrm{~m}, 2 \mathrm{H}), 7.16 \sim 7.12(\mathrm{~m}, 2 \mathrm{H}), 5.19(\mathrm{~s}, 2 \mathrm{H}) ;{ }^{19} \mathrm{~F}$ NMR $\left(471 \mathrm{MHz}, \mathrm{CDCl}_{3}\right) \delta:-111.15 \sim-111.21(\mathrm{~m}) ;{ }^{13} \mathrm{C}$ NMR $\left(126 \mathrm{MHz}, \mathrm{CDCl}_{3}\right) \delta: 163.95,163.06\left(\mathrm{~d},{ }^{1} J_{\mathrm{C}-\mathrm{F}}=\right.$ $250.6 \mathrm{~Hz}, \mathrm{ArF}), 159.61,158.10,149.13,148.57,134.51$, $131.68\left(\mathrm{~d},{ }^{3} J_{\mathrm{C}^{-} \mathrm{F}}=8.3 \mathrm{~Hz}, \mathrm{ArF}\right), 130.39,129.37,123.89$, $121.88,118.99,116.97,116.53\left(\mathrm{~d},{ }^{2} J_{\mathrm{C}-\mathrm{F}}=21.8 \mathrm{~Hz}, \mathrm{ArF}\right)$, 
109.21, 59.97; IR (KBr) v: 3095, 2940, 2861, 1612, 1526, $1343,1242,1118,854 \mathrm{~cm}^{-1}$. HRMS (ESI) calcd for $\mathrm{C}_{22} \mathrm{H}_{15} \mathrm{FN}_{3} \mathrm{O}_{6}\left[(\mathrm{M}+\mathrm{H})^{+}\right]:$: 436.0934, found 436.0939.

3-(4-硝基苯基)-4-(4-三氟甲基苯基)-5-(3-硝基苯氧 甲基)异噁唑(5-15)：白色固体, 产率 84\%. m.p. 132.2 $133.1{ }^{\circ} \mathrm{C} ;{ }^{1} \mathrm{H}$ NMR $\left(500 \mathrm{MHz}, \mathrm{CDCl}_{3}\right) \delta: 8.23(\mathrm{~d}, J=8.5$ $\mathrm{Hz}, 2 \mathrm{H}), 7.90(\mathrm{~d}, J=7.5 \mathrm{~Hz}, 1 \mathrm{H}), 7.76(\mathrm{~d}, J=2.0 \mathrm{~Hz}, 1 \mathrm{H})$, 7.70 (d, $J=7.5 \mathrm{~Hz}, 2 \mathrm{H}), 7.64 \sim 7.62(\mathrm{~m}, 2 \mathrm{H}), 7.49$ (t, $J=$ $8.0 \mathrm{~Hz}, 1 \mathrm{H}), 7.39$ (d, $J=7.5 \mathrm{~Hz}, 2 \mathrm{H}), 7.29$ (d, $J=8.0 \mathrm{~Hz}$, $1 \mathrm{H}), 5.22(\mathrm{~s}, 2 \mathrm{H}) ;{ }^{19} \mathrm{~F}$ NMR $\left(471 \mathrm{MHz}, \mathrm{CDCl}_{3}\right) \delta$ : $-62.82 ;{ }^{13} \mathrm{C} \mathrm{NMR}\left(126 \mathrm{MHz}, \mathrm{CDCl}_{3}\right) \delta: 164.34,159.61$, $157.97,149.22,148.76,134.11,131.77,131.34\left(\mathrm{q},{ }^{2} J_{\mathrm{C}-\mathrm{F}}=\right.$ $\left.33.1 \mathrm{~Hz}, \mathrm{CF}_{3}\right), 130.44,130.13,129.44,126.33\left(\mathrm{q},{ }^{3} J_{\mathrm{C}-\mathrm{F}}=\right.$ $\left.3.6 \mathrm{~Hz}, \mathrm{ArCF}_{3}\right), 124.06,123.67\left(\mathrm{q},{ }^{1} J_{\mathrm{C}-\mathrm{F}}=272.5 \mathrm{~Hz}\right.$, $\left.\mathrm{ArCF}_{3}\right), 121.87,118.72,117.20,109.10,59.96$; IR (KBr) $v$ : 2900, 1620, 1527, 1343, 1247, 1164, $855 \mathrm{~cm}^{-1}$. HRMS (ESI) calcd for $\mathrm{C}_{23} \mathrm{H}_{15} \mathrm{~F}_{3} \mathrm{~N}_{3} \mathrm{O}_{6}\left[(\mathrm{M}+\mathrm{H})^{+}\right]$: 486.0902, found 486.0907.

3-(4-硝基苯基)-4-苯基-5-(3-甲基苯氧甲基)异噁唑 (5-16): 白色固体, 产率 83\%. m.p. $99.8 \sim 100.3{ }^{\circ} \mathrm{C} ;{ }^{1} \mathrm{H}$ NMR (500 MHz, $\left.\mathrm{CDCl}_{3}\right) \delta: 8.21(\mathrm{~d}, J=9.0 \mathrm{~Hz}, 2 \mathrm{H}), 7.66$ (d, $J=9.0 \mathrm{~Hz}, 2 \mathrm{H}), 7.44 \sim 7.39$ (m, $3 \mathrm{H}), 7.22$ (dt, $J=6.0$, $2.0 \mathrm{~Hz}, 2 \mathrm{H}), 7.15(\mathrm{dd}, J=9.0,8.0 \mathrm{~Hz}, 1 \mathrm{H}), 6.83(\mathrm{~d}, J=7.5$ $\mathrm{Hz}, 1 \mathrm{H}), 6.75 \sim 6.74(\mathrm{~m}, 2 \mathrm{H}), 5.09$ (s, 2H), 2.31 (s, 3H); ${ }^{13} \mathrm{C}$ NMR $\left(126 \mathrm{MHz}, \mathrm{CDCl}_{3}\right) \delta: 165.09,159.51,157.79$, $148.50,139.80,135.00,129.83,129.43,129.39,129.20$, $128.87,128.27,123.82,122.77,119.43,115.69,111.84$, 59.47, 21.52; IR (KBr) v: 3085, 2918, 2860, 1597, 1520, $1346,1252,1166,1028,851 \mathrm{~cm}^{-1}$. HRMS (ESI) calcd for $\mathrm{C}_{23} \mathrm{H}_{19} \mathrm{~N}_{2} \mathrm{O}_{4}\left[(\mathrm{M}+\mathrm{H})^{+}\right]$: 387.1331, found 387.1338.

3-(4-硝基苯基)-4-(4-氟苯基)-5-(3-甲基苯氧甲基)异 噁唑(5-17): 白色固体, 产率 $83 \%$. m.p. 94.3 95.7 ${ }^{\circ} \mathrm{C}$; ${ }^{1} \mathrm{H}$ NMR (500 MHz, $\left.\mathrm{CDCl}_{3}\right) \delta: 8.22(\mathrm{dd}, J=8.0,2.0 \mathrm{~Hz}$, $2 \mathrm{H}), 7.65(\mathrm{~d}, J=9.0 \mathrm{~Hz}, 2 \mathrm{H}), 7.23 \sim 7.16(\mathrm{~m}, 3 \mathrm{H}), 7.12(\mathrm{t}$, $J=8.5 \mathrm{~Hz}, 2 \mathrm{H}), 6.84(\mathrm{~d}, J=7.5 \mathrm{~Hz}, 1 \mathrm{H}), 6.75 \sim 6.74(\mathrm{~m}$, $2 \mathrm{H}), 5.08$ (s, 2H), 2.32 (s, 3H); ${ }^{19} \mathrm{~F}$ NMR (471 MHz, $\left.\mathrm{CDCl}_{3}\right) \delta:-111.84 \sim 111.89(\mathrm{~m}) ;{ }^{13} \mathrm{C}$ NMR $(126 \mathrm{MHz}$, $\left.\mathrm{CDCl}_{3}\right) \delta: 165.24,162.96\left(\mathrm{~d},{ }^{1} J_{\mathrm{C}-\mathrm{F}}=250.0 \mathrm{~Hz}, \mathrm{ArF}\right)$, $159.51,157.72,148.55,139.86,134.80,131.69\left(\mathrm{~d},{ }^{3} J_{\mathrm{C}-\mathrm{F}}=\right.$ $8.2 \mathrm{~Hz}, \mathrm{ArF}), 129.43,129.40,124.26,123.89,122.88$, $118.47,116.36\left(\mathrm{~d},{ }^{2} J_{\mathrm{C}-\mathrm{F}}=21.4 \mathrm{~Hz}, \mathrm{ArF}\right), 116.28,115.70$, 111.80, 59.51, 21.50; IR (KBr) v: 3083, 2924, 2864, 1597, 1514, 1348, 1227, 1164, 1028, $848 \mathrm{~cm}^{-1}$. HRMS (EI) calcd for $\mathrm{C}_{23} \mathrm{H}_{18} \mathrm{FN}_{2} \mathrm{O}_{24}\left[(\mathrm{M}+\mathrm{H})^{+}\right]$: 405.1246, found 405.1245 .
3-(4-硝基苯基)-4-(4-三氟甲基苯基)-5-((3-甲基苯氧 甲基)异噁唑(5-18): 白色固体, 产率 85\%. m.p. 92.5 $93.3{ }^{\circ} \mathrm{C} ;{ }^{1} \mathrm{H}$ NMR (500 MHz, $\left.\mathrm{CDCl}_{3}\right) \delta: 8.23$ (dd, $J=7.0$, $2.0 \mathrm{~Hz}, 2 \mathrm{H}), 7.66(\mathrm{~d}, J=8.0 \mathrm{~Hz}, 2 \mathrm{H}), 7.63(\mathrm{dd}, J=7.0,2.0$ $\mathrm{Hz}, 2 \mathrm{H}), 7.36(\mathrm{~d}, J=8.0 \mathrm{~Hz}, 2 \mathrm{H}), 7.20(\mathrm{t}, J=7.5 \mathrm{~Hz}, 1 \mathrm{H})$, 6.85 (d, $J=8.0 \mathrm{~Hz}, 1 \mathrm{H}), 6.74$ (t, $J=7.5 \mathrm{~Hz}, 2 \mathrm{H}), 5.09$ (s, $2 \mathrm{H}), 2.31(\mathrm{~s}, 3 \mathrm{H}) ;{ }^{19} \mathrm{~F}$ NMR $\left(471 \mathrm{MHz}, \mathrm{CDCl}_{3}\right) \delta$ : -62.77. ${ }^{13} \mathrm{C}$ NMR $\left(126 \mathrm{MHz}, \mathrm{CDCl}_{3}\right) \delta: 165.73,159.52$, $157.59,148.67,139.93,134.46,132.18,130.95\left(\mathrm{q},{ }^{2} J_{\mathrm{C}^{-\mathrm{F}}}=\right.$ $\left.32.7 \mathrm{~Hz}, \mathrm{ArCF}_{3}\right), 130.20,129.47,126.10\left(\mathrm{q},{ }^{3} J_{\mathrm{C}-\mathrm{F}}=3.7\right.$ $\mathrm{Hz}, \mathrm{ArCF}_{3}$ ), 124.00, 123.81 (q, ${ }^{1} J_{\mathrm{C}-\mathrm{F}}=273.4 \mathrm{~Hz}, \mathrm{CF}_{3}$ ), $123.00,118.18,115.69,111.72,59.58,21.47$; IR (KBr) $v$ : 3074, 2928, 2863, 1598, 1521, 1333, 1260, 1117, 851 $\mathrm{cm}^{-1}$. HRMS (ESI) calcd for $\mathrm{C}_{24} \mathrm{H}_{18} \mathrm{~F}_{3} \mathrm{~N}_{2} \mathrm{O}_{4}\left[(\mathrm{M}+\mathrm{H})^{+}\right]$: 455.1217, found 455.1213 .

3-(3-甲氧基苯基)-4-苯基-5-(4-甲氧基苯氧甲基)异 噁唑(5-19): 白色固体, 产率 89\%. m.p. 104.5 105.4 ${ }^{\circ} \mathrm{C}$; ${ }^{1} \mathrm{H}$ NMR $\left(500 \mathrm{MHz}, \mathrm{CDCl}_{3}\right) \delta: 7.77(\mathrm{dd}, J=8.5,2.0 \mathrm{~Hz}$, $2 \mathrm{H}), 7.55(\mathrm{~d}, J=8.5 \mathrm{~Hz}, 2 \mathrm{H}), 7.42 \sim 7.38(\mathrm{~m}, 2 \mathrm{H}), 7.14 \sim$ $7.08(\mathrm{~m}, 1 \mathrm{H}), 7.04(\mathrm{~d}, J=8.5 \mathrm{~Hz}, 1 \mathrm{H}), 6.92(\mathrm{dd}, J=7.0$, $2.0 \mathrm{~Hz}, 2 \mathrm{H}), 6.84(\mathrm{dd}, J=7.0,5.0 \mathrm{~Hz}, 2 \mathrm{H}), 6.60(\mathrm{~s}, 1 \mathrm{H})$, 5.14 (s, 2H), 3.86 (s, 3H), 3.77 (s, 3H); ${ }^{19} \mathrm{~F}$ NMR (471 $\left.\mathrm{MHz}, \mathrm{CDCl}_{3}\right) \delta:-110.33 \sim-118.43(\mathrm{~m}) ;{ }^{13} \mathrm{C}$ NMR $(126$ $\left.\mathrm{MHz}, \mathrm{CDCl}_{3}\right) \delta: 168.67,162.10,158.03,154.70,152.03$, $137.81,131.28,129.62,129.48,128.18,127.42,127.36$, $121.51,116.10,114.84,111.58,101.01,62.30,56.38$, 55.70; IR (KBr) v: 3060, 2931, 2838, 1610, 1511, 1448, $1231,1030,815 \mathrm{~cm}^{-1}$. HRMS (ESI) calcd for $\mathrm{C}_{24} \mathrm{H}_{22} \mathrm{NO}_{4}$ $\left[(\mathrm{M}+\mathrm{H})^{+}\right]$: 388.1538 , found 388.1543 .

3-(3-甲氧基苯基)-4-(4-氟苯基)-5-(4-甲氧基苯氧甲 基)异噁唑(5-20): 白色固体, 产率 91\%. m.p. 101.3 $102.2{ }^{\circ} \mathrm{C} ;{ }^{1} \mathrm{H}$ NMR $\left(500 \mathrm{MHz}, \mathrm{CDCl}_{3}\right) \delta: 7.80(\mathrm{dd}, J=8.5$, $2.5 \mathrm{~Hz}, 1 \mathrm{H}), 7.74(\mathrm{~d}, J=2.0 \mathrm{~Hz}, 1 \mathrm{H}), 7.52$ (dd, $J=9.0,5.5$ $\mathrm{Hz}, 2 \mathrm{H}), 7.11$ (t, $J=9.0 \mathrm{~Hz}, 2 \mathrm{H}), 7.04(\mathrm{~d}, J=8.5 \mathrm{~Hz}, 1 \mathrm{H})$, $6.92(\mathrm{~d}, J=9.0 \mathrm{~Hz}, 2 \mathrm{H}), 6.85(\mathrm{~d}, J=9.0 \mathrm{~Hz}, 2 \mathrm{H}), 6.60$ (s, $1 \mathrm{H}), 5.14(\mathrm{~s}, 2 \mathrm{H}), 3.86(\mathrm{~s}, 3 \mathrm{H}), 3.77$ (s, 3H); ${ }^{19} \mathrm{~F}$ NMR (471 $\left.\mathrm{MHz}, \mathrm{CDCl}_{3}\right) \delta: 115.19 \sim 115.17(\mathrm{~m}) ;{ }^{13} \mathrm{C}$ NMR $(126$ $\left.\mathrm{MHz}, \mathrm{CDCl}_{3}\right) \delta: 168.71,162.20\left(\mathrm{~d},{ }^{1} J_{\mathrm{C}-\mathrm{F}}=246.9 \mathrm{~Hz}, \mathrm{ArF}\right)$, $161.99,157.87,154.68,151.96,133.63,131.19\left(\mathrm{~d},{ }^{3} J_{\mathrm{C}-\mathrm{F}}=\right.$ $7.8 \mathrm{~Hz}, \mathrm{ArF}), 130.23,129.31,127.40,121.53,116.06$, $115.02\left(\mathrm{~d},{ }^{2} J_{\mathrm{C}-\mathrm{F}}=21.3 \mathrm{~Hz}, \mathrm{ArF}\right), 114.80,111.49,101.09$, 62.36, 55.73, 55.69; IR (KBr) v: 3071, 2935, 2834, 1605, 1510, 1448, 1231, 1164, 1027, $814 \mathrm{~cm}^{-1}$. HRMS (ESI) calcd for $\mathrm{C}_{24} \mathrm{H}_{21} \mathrm{FNO}_{3}\left[(\mathrm{M}+\mathrm{H})^{+}\right]$: 406.1443, found 406.1449 . 
3-(3-甲氧基苯基)-4-(4-三氟甲基苯基)-5-(4-甲氧基 苯氧甲基)异噁唑(5-21): 白色固体，产率 88\%. m.p. $114.6 \sim 114.8{ }^{\circ} \mathrm{C} ;{ }^{1} \mathrm{H}$ NMR $\left(500 \mathrm{MHz}, \mathrm{CDCl}_{3}\right) \delta: 7.81$ (dd, $J=8.5,2.5 \mathrm{~Hz}, 1 \mathrm{H}), 7.76(\mathrm{~d}, J=2.5 \mathrm{~Hz}, 1 \mathrm{H}), 7.65(\mathrm{dd}, J=$ 9.0, $2.5 \mathrm{~Hz}, 4 \mathrm{H}), 7.07$ (d, $J=9.0 \mathrm{~Hz}, 1 \mathrm{H}), 6.92$ (d, $J=9.0$ $\mathrm{Hz}, 2 \mathrm{H}), 6.85$ (d, $J=9.0 \mathrm{~Hz}, 2 \mathrm{H}), 6.61$ (s, 1H), 5.15 (s, $2 \mathrm{H}), 3.88(\mathrm{~s}, 3 \mathrm{H}), 3.78(\mathrm{~s}, 3 \mathrm{H}) ;{ }^{19} \mathrm{~F} \mathrm{NMR}(471 \mathrm{MHz}$, $\left.\mathrm{CDCl}_{3}\right) \delta$ : $-62.46 ;{ }^{13} \mathrm{C} \mathrm{NMR}\left(126 \mathrm{MHz}, \mathrm{CDCl}_{3}\right) \delta$ : $168.82,161.85,157.87,154.69,151.93,129.88,129.80$, $129.33\left(\mathrm{q},{ }^{2} J_{\mathrm{C}-\mathrm{F}}=32.4 \mathrm{~Hz}, \mathrm{ArCF}_{3}\right), 129.32,128.09,125.01$ $\left(\mathrm{q},{ }^{3} J_{\mathrm{C}-\mathrm{F}}=3.6 \mathrm{~Hz}, \mathrm{ArCF}_{3}\right), 124.30\left(\mathrm{q},{ }^{1} J_{\mathrm{C}-\mathrm{F}}=273.4 \mathrm{~Hz}\right.$, $\left.\mathrm{ArCF}_{3}\right), 123.22,121.68,116.05,114.80,111.60,101.04$, 62.37, 55.77, 55.70; IR (KBr) v: 2946, 2840, 1612, 1511, 1453, 1323, 1234, 1123, 1025, $814 \mathrm{~cm}^{-1}$. HRMS (EI) calcd for $\mathrm{C}_{25} \mathrm{H}_{21} \mathrm{~F}_{3} \mathrm{NO}_{4}\left[(\mathrm{M}+\mathrm{H})^{+}\right]$: 456.1408, found 456.1417 .

3-(3-甲氧基苯基)-4-苯基-5-(3-硝基苯氧甲基)异噁 唑(5-22): 白色固体, 产率 $85 \%$. m.p. $158.9 \sim 159.4^{\circ} \mathrm{C}$; ${ }^{1} \mathrm{H}$ NMR $\left(500 \mathrm{MHz}, \mathrm{CDCl}_{3}\right) \delta: 7.90 \sim 7.88(\mathrm{~m}, 1 \mathrm{H}), 7.85$ (t, $J=2.5 \mathrm{~Hz}, 1 \mathrm{H}), 7.79$ (dd, $J=8.5,2.5 \mathrm{~Hz}, 1 \mathrm{H}), 7.75$ (d, $J=2.0 \mathrm{~Hz}, 1 \mathrm{H}), 7.55 \sim 7.53(\mathrm{~m}, 2 \mathrm{H}), 7.48(\mathrm{t}, J=8.0 \mathrm{~Hz}$, $1 \mathrm{H}), 7.44 \sim 7.41(\mathrm{~m}, 2 \mathrm{H}), 7.38 \sim 7.34(\mathrm{~m}, 1 \mathrm{H}), 7.33 \sim 7.31$ $(\mathrm{m}, 1 \mathrm{H}), 7.06(\mathrm{~d}, J=8.5 \mathrm{~Hz}, 1 \mathrm{H}), 6.67(\mathrm{~s}, 1 \mathrm{H}), 5.28(\mathrm{~s}$, $2 \mathrm{H}), 3.87(\mathrm{~s}, 3 \mathrm{H}) ;{ }^{13} \mathrm{C} \mathrm{NMR}\left(126 \mathrm{MHz}, \mathrm{CDCl}_{3}\right) \delta: 166.90$, $162.20,158.25,158.11,149.23,137.67,131.31,130.36$, $129.54,129.47,128.14,127.41,127.30,121.63,121.14$, $116.87,111.55,109.43,101.82,61.69,55.75$; IR (KBr) $v$ : 3106, 2993,2940, 2840, 1609, 1528, 1350, 1242, 1056, 813 $\mathrm{cm}^{-1}$. HRMS (ESI) calcd for $\mathrm{C}_{23} \mathrm{H}_{19} \mathrm{~N}_{2} \mathrm{O}_{5}\left[(\mathrm{M}+\mathrm{H})^{+}\right]$: 403.1291, found 403.1288.

3-(3-甲氧基苯基)-4-(4-氟苯基)-5-(3-硝基苯氧甲基) 异噁唑(5-23): 白色固体，产率 85\%. m.p. 166.2 $167.3{ }^{\circ} \mathrm{C} ;{ }^{1} \mathrm{H}$ NMR $\left(500 \mathrm{MHz}, \mathrm{CDCl}_{3}\right) \delta: 7.90$ (ddd, $J=$ 8.0, 2.0, $1.0 \mathrm{~Hz}, 1 \mathrm{H}), 7.85$ (t, $J=2.5 \mathrm{~Hz}, 1 \mathrm{H}), 7.78$ (dd, $J=$ $8.5,2.5 \mathrm{~Hz}, 1 \mathrm{H}), 7.75(\mathrm{~d}, J=8.5 \mathrm{~Hz}, 1 \mathrm{H}), 7.55(\mathrm{~m}, 3 \mathrm{H})$, 7.35 (dt, $J=8.0,2.0 \mathrm{~Hz}, 1 \mathrm{H}), 7.12(\mathrm{dd}, J=8.5,2.0 \mathrm{~Hz}$, $2 \mathrm{H}), 7.05(\mathrm{~d}, J=8.5 \mathrm{~Hz}, 1 \mathrm{H}), 6.67(\mathrm{~s}, 1 \mathrm{H}), 5.28(\mathrm{~s}, 2 \mathrm{H})$, $3.87(\mathrm{~s}, 3 \mathrm{H}) ;{ }^{19} \mathrm{~F}$ NMR $\left(471 \mathrm{MHz}, \mathrm{CDCl}_{3}\right) \delta:-115.03 \sim$ $-115.09(\mathrm{~m}) ;{ }^{13} \mathrm{C} \mathrm{NMR}\left(126 \mathrm{MHz}, \mathrm{CDCl}_{3}\right) \delta: 166.93$, $162.24\left(\mathrm{~d},{ }^{1} J_{\mathrm{C}-\mathrm{F}}=246.9 \mathrm{~Hz}\right), 162.12,158.23,158.01$, 149.26, 133.52, $131.15\left(\mathrm{~d},{ }^{3} J_{\mathrm{C}-\mathrm{F}}=7.6 \mathrm{~Hz}, \mathrm{ArF}\right), 130.36$, $130.34,129.32,127.40,121.65,121.19,116.93,115.03$ (d, $\left.{ }^{2} J_{\mathrm{C}-\mathrm{F}}=21.4 \mathrm{~Hz}, \mathrm{ArF}\right), 111.52,109.40,101.75,61.72$, 55.76; IR (KBr) v: 3097, 2918, 2851, 1611, 1529, 1355, 1237, 1164, 1055, $825 \mathrm{~cm}^{-1}$. HRMS (ESI) calcd for
$\mathrm{C}_{23} \mathrm{H}_{18} \mathrm{FN}_{2} \mathrm{O}_{5}\left[(\mathrm{M}+\mathrm{H})^{+}\right]:$421.1194, found 421.1194 .

3-(3-甲氧基苯基)-4-(4-三氟甲基苯基)-5-(3-硝基苯 氧甲基) 异噁唑(5-24): 白色固体，产率 82\%. m.p. $157.8 \sim 158.0{ }^{\circ} \mathrm{C} ;{ }^{1} \mathrm{H}$ NMR $\left(500 \mathrm{MHz}, \mathrm{CDCl}_{3}\right) \delta: 7.91(\mathrm{dd}$, $J=8.0,1.0 \mathrm{~Hz}, 1 \mathrm{H}), 7.85(\mathrm{t}, J=2.5 \mathrm{~Hz}, 1 \mathrm{H}), 7.82(\mathrm{dd}, J=$ 8.0, $2.0 \mathrm{~Hz}, 1 \mathrm{H}), 7.67(\mathrm{~d}, J=2.0 \mathrm{~Hz}, 1 \mathrm{H}), 7.71 \sim 7.63(\mathrm{~m}$, 4H), $7.49(\mathrm{t}, J=8.0 \mathrm{~Hz}, 1 \mathrm{H}), 7.32(\mathrm{dd}, J=8.5,2.0 \mathrm{~Hz}$, $1 \mathrm{H}), 7.08$ (d, $J=8.5 \mathrm{~Hz}, 1 \mathrm{H}), 6.67(\mathrm{~s}, 1 \mathrm{H}), 5.29(\mathrm{~s}, 2 \mathrm{H})$, $3.88(\mathrm{~s}, 3 \mathrm{H}) ;{ }^{19} \mathrm{~F}$ NMR $\left(471 \mathrm{MHz}, \mathrm{CDCl}_{3}\right) \delta:-62.39 ;{ }^{13} \mathrm{C}$ NMR (126 MHz, $\left.\mathrm{CDCl}_{3}\right) \delta: 167.07,161.97,158.22$, $158.00,149.23,141.33,130.37,129.87,129.85,129.33$ (q, $\left.{ }^{2} J_{\mathrm{C}-\mathrm{F}}=32.4 \mathrm{~Hz}, \mathrm{ArCF}_{3}\right), 129.28,128.12,124.30$ (q, $\left.{ }^{1} J_{\mathrm{C}-\mathrm{F}}=272.2 \mathrm{~Hz}, \mathrm{CF}_{3}\right), 125.01\left(\mathrm{q},{ }^{3} J_{\mathrm{C}-\mathrm{F}}=3.8 \mathrm{~Hz}, \mathrm{ArCF}_{3}\right)$, $121.63,121.36,116.90,111.66,109.38,101.74,61.68$, 55.78; IR (KBr) v: 2932, 2849, 1612, 1524, 1330, 1246, 1119, 1068, $825 \mathrm{~cm}^{-1}$. HRMS (ESI) calcd for $\mathrm{C}_{24} \mathrm{H}_{18} \mathrm{~F}_{3} \mathrm{~N}_{2} \mathrm{O}_{5}\left[(\mathrm{M}+\mathrm{H})^{+}\right]$: 471.1160, found 471.1162.

3-(3-甲氧基苯基)-4-苯基-5-(4-氟苯氧甲基)异啞唑 (5-25): 白色固体，产率 90\%. m.p. 121.5 122.1 ${ }^{\circ} \mathrm{C} ;{ }^{1} \mathrm{H}$ NMR (500 MHz, $\left.\mathrm{CDCl}_{3}\right) \delta: 7.79(\mathrm{~d}, J=8.5 \mathrm{~Hz}, 1 \mathrm{H}), 7.75$ $(\mathrm{d}, J=2.0 \mathrm{~Hz}, 1 \mathrm{H}), 7.55$ (d, $J=8.5 \mathrm{~Hz}, 2 \mathrm{H}), 7.44$ (t, $J=8.0$ $\mathrm{Hz}, 2 \mathrm{H}), 7.35$ (d, $J=8.5 \mathrm{~Hz}, 1 \mathrm{H}), 7.05$ (d, $J=8.5 \mathrm{~Hz}, 1 \mathrm{H})$, $7.01(\mathrm{~d}, J=8.5 \mathrm{~Hz}, 2 \mathrm{H}), 6.96 \sim 6.91(\mathrm{~m}, 2 \mathrm{H}), 6.61(\mathrm{~s}, 1 \mathrm{H})$, $5.16(\mathrm{~s}, 2 \mathrm{H}), 3.87(\mathrm{~s}, 3 \mathrm{H}) ;{ }^{19} \mathrm{~F}$ NMR (471 MHz, $\left.\mathrm{CDCl}_{3}\right) \delta$ : $-122.28 \sim-122.34(\mathrm{~m}) ;{ }^{13} \mathrm{C} \mathrm{NMR}\left(126 \mathrm{MHz}, \mathrm{CDCl}_{3}\right) \delta$ : $168.11,162.13,158.03,157.91\left(\mathrm{~d},{ }^{1} J_{\mathrm{C}-\mathrm{F}}=240.5 \mathrm{~Hz}, \mathrm{ArF}\right)$, $153.94,137.72,131.30,129.54,129.51,128.13,127.38$, 127.27, 121.34, $116.13\left(\mathrm{~d},{ }^{2} J_{\mathrm{C}-\mathrm{F}}=23.7 \mathrm{~Hz}, \mathrm{ArF}\right), 116.11$ $\left(\mathrm{d},{ }^{3} J_{\mathrm{C}-\mathrm{F}}=7.9 \mathrm{~Hz}, \mathrm{ArF}\right), 111.50,101.29,100.00,62.18$, 55.74; IR (KBr) v: 3063, 2936, 2840, 1608, 1508, 1446, 1365, 1241, 1025, $817 \mathrm{~cm}^{-1}$. HRMS (ESI) calcd for $\mathrm{C}_{23} \mathrm{H}_{19} \mathrm{FNO}_{3}\left[(\mathrm{M}+\mathrm{H})^{+}\right]: 376.1338$, found 376.1343.

3-(3-甲氧基苯基)-4-(4-氟苯基)-5-(4-氟苯氧甲基)异 啞唑(5-26): 白色固体，产率 88\%. m.p. 105.8 106.1 ${ }^{\circ} \mathrm{C}$; ${ }^{1} \mathrm{H} \mathrm{NMR}\left(500 \mathrm{MHz}, \mathrm{CDCl}_{3}\right) \delta: 7.77(\mathrm{dd}, J=8.5,2.5 \mathrm{~Hz}$, $1 \mathrm{H}), 7.73(\mathrm{~d}, J=2.0 \mathrm{~Hz}, 1 \mathrm{H}), 7.54 \sim 7.48(\mathrm{~m}, 2 \mathrm{H}), 7.11(\mathrm{dt}$, $J=7.0,2.0 \mathrm{~Hz}, 2 \mathrm{H}), 7.05(\mathrm{~d}, J=8.5 \mathrm{~Hz}, 1 \mathrm{H}), 7.03 \sim 6.98$ $(\mathrm{m}, 2 \mathrm{H}), 6.95 \sim 6.92(\mathrm{~m}, 2 \mathrm{H}), 6.61(\mathrm{~s}, 1 \mathrm{H}), 5.16(\mathrm{~s}, 2 \mathrm{H})$, $3.87(\mathrm{~s}, 3 \mathrm{H}) ;{ }^{19} \mathrm{~F}$ NMR (471 MHz, DMSO) $\delta:-115.12 \sim$ $-115.15(\mathrm{~m}),-122.76 \sim-122.81(\mathrm{~m}) ;{ }^{13} \mathrm{C}$ NMR $(126$ $\mathrm{MHz}, \mathrm{DMSO}) \delta: 168.63,161.98\left(\mathrm{~d},{ }^{1} J_{\mathrm{C}-\mathrm{F}}=244.9 \mathrm{~Hz}\right.$, ArF), 161.97, 158.42, $157.48\left(\mathrm{~d},{ }^{1} J_{\mathrm{C}^{-\mathrm{F}}}=237.5 \mathrm{~Hz}, \mathrm{ArF}\right)$, 154.36, 134.10, 134.08, $131.77\left(\mathrm{~d},{ }^{3} J_{\mathrm{C}^{-}-\mathrm{F}}=8.07 \mathrm{~Hz}, \mathrm{ArF}\right)$, $129.80,129.15,127.93,121.40,116.73\left(\mathrm{~d},{ }^{3} J_{\mathrm{C}-\mathrm{F}}=8.15 \mathrm{~Hz}\right.$, ArF), $116.43\left(\mathrm{~d},{ }^{2} J_{\mathrm{C}^{-\mathrm{F}}}=23.09 \mathrm{~Hz}, \operatorname{ArF}\right), 115.33\left(\mathrm{~d},{ }^{2} J_{\mathrm{C}-\mathrm{F}}=\right.$ 
$21.3 \mathrm{~Hz}, \mathrm{ArF}), 112.71,102.72,61.56,56.20$; IR (KBr) v: 3066, 2956, 2843, 1610, 1512, 1450, 1357, 1220, 1069, $823 \mathrm{~cm}^{-1}$. HRMS (ESI) calcd for $\mathrm{C}_{23} \mathrm{H}_{18} \mathrm{~F}_{2} \mathrm{NO}_{3}\left[(\mathrm{M}+\mathrm{H})^{+}\right]$: 394.1243, found 394.1249.

3-(3-甲氧基苯基)-4-(4-三氟甲基苯基)-5-(4-氟苯氧 甲基)异噁唑(5-27): 白色固体, 产率 82\%. m.p. 108.0 $108.7{ }^{\circ} \mathrm{C} ;{ }^{1} \mathrm{H}$ NMR $\left(500 \mathrm{MHz}, \mathrm{CDCl}_{3}\right) \delta: 7.82$ (dd, $J=8.0$, $2.0 \mathrm{~Hz}, 1 \mathrm{H}), 7.76(\mathrm{~d}, J=2.0 \mathrm{~Hz}, 1 \mathrm{H}), 7.68(\mathrm{~d}, J=8.5 \mathrm{~Hz}$, 2H), 7.65 (d, $J=8.5 \mathrm{~Hz}, 2 \mathrm{H}), 7.08$ (d, $J=8.5 \mathrm{~Hz}, 1 \mathrm{H}), 7.02$ $(\mathrm{t}, J=9.0 \mathrm{~Hz}, 2 \mathrm{H}), 6.94(\mathrm{dd}, J=9.0,4.0 \mathrm{~Hz}, 2 \mathrm{H}), 6.62$ (s, $1 \mathrm{H}), 5.17(\mathrm{~s}, 2 \mathrm{H}), 3.88(\mathrm{~s}, 3 \mathrm{H}) ;{ }^{19} \mathrm{~F}$ NMR (471 MHz, $\left.\mathrm{CDCl}_{3}\right) \delta:-62.46(\mathrm{~s}),-122.22 \sim-122.27(\mathrm{~m}) ;{ }^{13} \mathrm{C}$ NMR (126 MHz, $\left.\mathrm{CDCl}_{3}\right) \delta: 168.30,161.90,157.94$, $157.90\left(\mathrm{~d},{ }^{1} J_{\mathrm{C}-\mathrm{F}}=239.7 \mathrm{~Hz}\right), 153.95,129.90,129.31$ (q, $\left.{ }^{2} J=32.3 \mathrm{~Hz}, \mathrm{ArCF}_{3}\right), 129.28,128.12,125.01\left(\mathrm{q},{ }^{3} J_{\mathrm{C}-\mathrm{F}}=\right.$ $3.7 \mathrm{~Hz}, \mathrm{ArCF}_{3}$ ), 124.35 (q, $\left.{ }^{1} J_{\mathrm{C}-\mathrm{F}}=272.0 \mathrm{~Hz}, \mathrm{CF}_{3}\right), 121.58$, $116.13\left(\mathrm{~d},{ }^{2} J_{\mathrm{C}-\mathrm{F}}=23.2 \mathrm{~Hz}, \mathrm{ArF}\right), 116.08\left(\mathrm{~d},{ }^{3} J_{\mathrm{C}-\mathrm{F}}=8.1 \mathrm{~Hz}\right)$, 111.63, 101.23, 62.10, 55.74; IR (KBr) v: 3058, 2941, 2844, 1613, 1513, 1430, 1357, 1259, 1103, $830 \mathrm{~cm}^{-1}$. HRMS (ESI) calcd for $\mathrm{C}_{24} \mathrm{H}_{18} \mathrm{~F}_{4} \mathrm{NO}_{3}\left[(\mathrm{M}+\mathrm{H})^{+}\right]$: 444.1225 , found 444.1217.

3-(3-甲氧基苯基)-4-苯基-5-(3-甲基苯氧甲基)异噁 唑(5-28): 白色固体, 产率 91\%. m.p. 102.6 103.9 ${ }^{\circ} \mathrm{C}$; ${ }^{1} \mathrm{H}$ NMR $\left(500 \mathrm{MHz}, \mathrm{CDCl}_{3}\right) \delta: 7.79(\mathrm{dd}, J=8.5,2.0 \mathrm{~Hz}$, $1 \mathrm{H}), 7.75(\mathrm{~d}, J=2.5 \mathrm{~Hz}, 1 \mathrm{H}), 7.55(\mathrm{dd}, J=8.5,1.0 \mathrm{~Hz}$, 2H), 7.43 (t, $J=7.5 \mathrm{~Hz}, 2 \mathrm{H}), 7.35$ (t, $J=7.5 \mathrm{~Hz}, 1 \mathrm{H}), 7.20$ $(\mathrm{t}, J=8.0 \mathrm{~Hz}, 1 \mathrm{H}), 7.05(\mathrm{~d}, J=8.5 \mathrm{~Hz}, 1 \mathrm{H}), 6.83$ (d, $J=8.0$ $\mathrm{Hz}, 3 \mathrm{H}), 6.85 \sim 6.77(\mathrm{~m}, 1 \mathrm{H}), 5.19(\mathrm{~s}, 2 \mathrm{H}), 3.87(\mathrm{~s}, 3 \mathrm{H})$, $2.35(\mathrm{~s}, 3 \mathrm{H}) ;{ }^{13} \mathrm{C} \mathrm{NMR}\left(126 \mathrm{MHz}, \mathrm{CDCl}_{3}\right) \delta: 168.11$, $162.13,158.85,158.03,156.94,153.94,137.72,131.30$, $129.54,129.51,128.13,127.38,127.27,121.34,116.22$, $116.14,116.08,116.04,111.50,101.29,62.18,55.74$; IR (KBr) v: 3052, 2924, 2849, 1601, 1508, 1448, 1349, 1257, $1174,776 \mathrm{~cm}^{-1}$. HRMS (ESI) calcd for $\mathrm{C}_{24} \mathrm{H}_{22} \mathrm{NO}_{3}[(\mathrm{M}+$ $\mathrm{H})^{+}$]: 372.1587 , found 372.1594 .

3-(3-甲氧基苯基)-4-(4-氟苯基)-5-(3-甲基苯氧甲基) 异啞唑(5-29): 白色固体，产率 84\%. m.p. 103.8 $104.3{ }^{\circ} \mathrm{C} ;{ }^{1} \mathrm{H}$ NMR $\left(500 \mathrm{MHz}, \mathrm{CDCl}_{3}\right) \delta: 7.78$ (dd, $J=8.5$, $2.5 \mathrm{~Hz}, 1 \mathrm{H}), 7.73(\mathrm{~d}, J=2.0 \mathrm{~Hz}, 1 \mathrm{H}), 7.52$ (dd, $J=9.0,5.0$ $\mathrm{Hz}, 2 \mathrm{H}), 7.20$ (t, $J=7.5 \mathrm{~Hz}, 1 \mathrm{H}), 7.11(\mathrm{t}, J=8.5 \mathrm{~Hz}, 2 \mathrm{H})$, $7.04(\mathrm{~d}, J=8.5 \mathrm{~Hz}, 1 \mathrm{H}), 6.85 \sim 6.78(\mathrm{~m}, 3 \mathrm{H}), 6.62(\mathrm{~s}, 1 \mathrm{H})$, $5.18(\mathrm{~s}, 2 \mathrm{H}), 3.87(\mathrm{~s}, 3 \mathrm{H}), 2.36(\mathrm{~s}, 3 \mathrm{H}) ;{ }^{19} \mathrm{~F}$ NMR (471 $\left.\mathrm{MHz}, \mathrm{CDCl}_{3}\right) \delta:-115.06 \sim-115.11(\mathrm{~m}) ;{ }^{13} \mathrm{C}$ NMR $(126$ $\left.\mathrm{MHz}, \mathrm{CDCl}_{3}\right) \delta: 168.62,162.22\left(\mathrm{~d},{ }^{1} J_{\mathrm{C}-\mathrm{F}}=246.9 \mathrm{~Hz}, \mathrm{ArF}\right)$, $162.01,157.87,157.85,139.86,133.61,131.17\left(\mathrm{~d},{ }^{3} J_{\mathrm{C}-\mathrm{F}}=\right.$
$7.6 \mathrm{~Hz}, \mathrm{ArF}), 130.25,129.41,129.34,127.39,122.72$, 121.52, 115.67, $115.01\left(\mathrm{~d},{ }^{2} J_{\mathrm{C}-\mathrm{F}}=21.4 \mathrm{~Hz}, \mathrm{ArF}\right), 111.57$, $111.47,101.02,61.48,55.75,21.54$; IR (KBr) v: 3023, 2920, 2849, 1605, 1509, 1453, 1259, 1157, $785 \mathrm{~cm}^{-1}$. HRMS (ESI) calcd for $\mathrm{C}_{24} \mathrm{H}_{21} \mathrm{FNO}_{3}\left[(\mathrm{M}+\mathrm{H})^{+}\right]$: 390.1494 , found 390.1500 .

3-(3-甲氧基苯基)-4-(4-三氟甲基苯基) -5-(3-甲基苯 氧甲基) 异噁唑(5-30): 白色固体，产率 $86 \%$. m.p. 109.0 109.1 ${ }^{\circ} \mathrm{C} ;{ }^{1} \mathrm{H}$ NMR (500 MHz, $\left.\mathrm{CDCl}_{3}\right) \delta: 7.82(\mathrm{dd}$, $J=8.5,2.0 \mathrm{~Hz}, 1 \mathrm{H}), 7.77(\mathrm{~d}, J=2.0 \mathrm{~Hz}, 1 \mathrm{H}), 7.71 \sim 7.64$ $(\mathrm{m}, 4 \mathrm{H}), 7.20$ (t, $J=7.5 \mathrm{~Hz}, 1 \mathrm{H}), 7.07$ (d, $J=8.5 \mathrm{~Hz}, 1 \mathrm{H})$, $6.84 \sim 6.78(\mathrm{~m}, 3 \mathrm{H}), 6.62(\mathrm{~s}, 1 \mathrm{H}), 5.19(\mathrm{~s}, 2 \mathrm{H}), 3.88(\mathrm{~s}$, $3 \mathrm{H}), 2.35$ (s, 3H); ${ }^{19} \mathrm{~F}$ NMR (471 MHz, DMSO) $\delta$ : -61.12; ${ }^{13} \mathrm{C}$ NMR (126 MHz, DMSO- $\left.d_{6}\right) \delta: 168.92$, $161.86,158.11,158.03,141.99,139.67,130.56,129.74$, $129.27,129.23,128.65,128.25\left(\mathrm{q},{ }^{2} J_{\mathrm{C}-\mathrm{F}}=31.5 \mathrm{~Hz}\right.$, $\left.\mathrm{ArCF}_{3}\right), 125.26\left(\mathrm{q},{ }^{3} J_{\mathrm{C}-\mathrm{F}}=3.6 \mathrm{~Hz}, \mathrm{ArCF}_{3}\right), 124.82(\mathrm{q}$, $\left.{ }^{1} J_{\mathrm{C}-\mathrm{F}}=272.2 \mathrm{~Hz}, \mathrm{CF}_{3}\right), 122.63,121.63,115.90,112.74$, $112.09,102.50,60.87,56.15,21.43$; IR (KBr) v: 3012, 2928, 2849, 1615, 1456, 1326, 1260, 1117, $842 \mathrm{~cm}^{-1}$. HRMS (ESI) calcd for $\mathrm{C}_{25} \mathrm{H}_{21} \mathrm{~F}_{3} \mathrm{NO}_{3}\left[(\mathrm{M}+\mathrm{H})^{+}\right]$: 440.1462 , found 440.1468 .

辅助材料(Supporting Information) 所合成化合物的 ${ }^{1} \mathrm{H}$ NMR、 ${ }^{13} \mathrm{C}$ NMR 及含氟化合物的 ${ }^{19} \mathrm{~F} \mathrm{NMR}$ 谱图. 这 些材料可以免费从本刊网站(http://sioc-journal.cn/)上下 载.

\section{References}

[1] Castellano, S.; Kuck, D.; Viviano, M.; Yoo, J.; Lopeź-Vallejo, F.; Conti, P.; Tamborini, L.; Pinto, A.; Medina-Franco, J. L.; Sbardella, G. J. Med. Chem. 2011, 54, 7663.

[2] Kumar, K. A.; Jayaroopa, P. Chem. Biol. Sci. 2013, 3, 294.

[3] Dong, K. Y.; Qin, H. T.; Bao, X. X.; Liu, F.; Zhu, C. Org. Lett. 2014, 16, 5266.

[4] Pereź, J. M.; Ramon, D. J. ACS Sustainable Chem. Eng. 2015, 3, 2343.

[5] Fan, Y. J. Agrochem. Res. Appl. 2010, 14, 1 (in Chinese) (范玉杰, 农药研究与应用, 2010, 14, 1.)

[6] He, H. W.; Li, M. Q.; Huang, G. L. Agrochemicals 2000, 39, 4 (in Chinese) (贺红武，李美强，黄刚良，农药, 2000, 39, 4.)

[7] Eccles, S. A.; Massey, A.; Raynaud, F. I.; Sharp, S. Y.; Box, G.; Valenti, M.; Patterson, L.; Brandon, A. D. H.; Gowan, S.; Boxall, F.; Aherne, W.; Rowlands, M.; Hayes, A.; Martins, V.; Urban, F.; Boxall, K.; Prodromou, C.; Pearl, L.; James, K.; Matthews, T. P.; Cheung, K. M.; Kalusa, A.; Jones, K.; McDonald, E.; Barril, X.; Brough, P. A.; Cansfield, J. E.; Dymock, B.; Drysdale, M. J.; Finch, H.; Howes, R.; Hubbard, R. E.; Surgenor, A.; Webb, P.; Wood, M.; Wright, L.; Workman, P. Cancer Res. 2008, 68, 2850.

[8] Sun, R.; Li, Y.; Xiong, L.; Liu, Y.; Wang, Q. J. Agric. Food Chem. 2011, 59, 4851.

[9] Sperry, J.; Wright, D. Curr. Opin. Drug Discovery Dev. 2005, 8, 723. 
[10] Lee, Y.; Koyama, Y.; Yonekawa, M.; Tanaka, T. Macromolecules 2009, 42, 7709 .

[11] Masafumi, U.; Yuki, I.; Aoi, S.; Yuta, I.; Maiko, K.; Hiroko, S.; Tetsuya, M.; Takeaki, N.; Okiko, M. Tetrahedron 2011, 67, 4612.

[12] Zhou, Z. W.; Yan, J. F.; Tang, X. M. Chin. J. Org. Chem. 2010, 30, 582.

[13] Changtam, C.; Hongmanee, P.; Suksamrarn, A. Eur. J. Med. Chem. 2010, 45, 4446 .

[14] John, J. T.; David, L. B.; Jeffery, S. C.; Matthew, J. G.; Carol, M. K.; Jaime, L. M.; William, E. P.; Roland, S. R.; Alexander, F. S.; Zhang, Y. Y.; Ben, S. Z.; Karen, S. J. Med. Chem. 2000, 43, 777.

[15] Yermolina, M. V.; Wang, J.; Caffrey, M. J. Med. Chem. 2011, 54, 765.

[16] Jensen, A. A.; Plath, N.; Pedersen, M. H. F.; Isberg, V.; Krall., J. J. Med. Chem. 2013, 56, 1211.

[17] Vitale, P.; Tacconelli, S.; Perrone, M. G. J. Med. Chem. 2013, 56, 4277.

[18] Fournier, P. A.; Arbour, M.; Cauchon, E. Bioorg. Med. Chem. Lett. 2012, 22, 2670 .

[19] Gomha, S. M.; Badrey, M. G.; Abdalla, M. M.; Arafa, R. K. Med. Chem. Commun. 2014, 5, 1685.

[20] Crocker, C. E.; Khan, S.; Cameron, M. D.; Robertson, H. A.; Robertson, G. S.; Lograsso, P. ACS Chem. Neurosci. 2011, 2, 207.

[21] Chambers, J. W.; Pachori, A.; Howard, S.; Ganno, M.; Hansen, D.; Kamenecka, T.; Song, X.; Duckett, D.; Chen, W.; Ling, Y. Y.; Cherry, L.; Cameron, M. D.; Lin, L.; Ruiz, C. H.; Lograsso, P. ACS Chem. Neurosci. 2011, 2, 198.

[22] Kumar, D. J. S.; Ho, M. M.; Leung, J. M.; Toyokuni, T. Adv. Synth. Catal. 2002, 344, 1146.

[23] Sahoo, A. K.; Oda, T.; Nakao, Y.; Hiyama, T. Adv. Synth. Catal. 2004, 346, 1715.

[24] Fu, W. C.; Zhou, Z. Y.; Kwong, F. Y. Org. Chem. Front. 2016, 3, 273.

[25] Velcicky, J.; Soicke, A.; Steiner, R.; Schmalz, H. G. J. Am. Chem.
Soc. 2011, 133, 6948.

[26] Oberli, M. A.; Buchwald, S. L. Org. Lett. 2012, 14, 4606.

[27] Scott, P. J. L.; Clarke, A.; Richardson, J. Org. Lett. 2015, 17, 476.

[28] Yang, Y.; Oldenhuis, N. J.; Buchwald, S. L. Angew. Chem. Int. Ed. 2013, 52, 615 .

[29] Haag, B. A.; Sämann, C.; Jana, A.; Knochel, P. Angew. Chem. Int. Ed. 2011, 50, 7290.

[30] Piller, F. M.; Metzger, A.; Schade, M..; Haag, B. A.; Garyushin, A.; Knochel, P. Chem. Eur. J. 2009, 15, 7192.

[31] Bernhardt, S.; Manolikakes, G.; Kunz, T.; Knochel, P. Angew. Chem. Int. Ed. 2011, 50, 9205.

[32] (a) Rogers, R. S.; Talley, J. J.; Brown, D. L.; Nagarajan, S.; Carter, J. S.; Weier, R. M.; Stealey, Michael A.; Collins, Paul W.; Seibert, K. WO 9625405, 1996 [Chem. Abstr. 1996, 125, 247800].

(b) Rogers, R. S.; Talley, J. J.; Sikorski, J. A.; Devadas, B.; Graneto, M. J.; Carter, J. S.; Norman, B. H.; Lu, H. F; Brown, D. L.; Nagarajan, S. WO 9638442, 1996 [Chem. Abstr. 1997, 126, 104081].

[33] Yang, Y. D.; Zhang, M.; Zhu, Y. W.; Zhang, L.; Xie, Q. Q.; Song, L. P.; Deng, H. M. Chin. J. Chem. 2013, 31, 950.

[34] Xu, S. S.; Zhang, M.; Zhang, L.; Jiang, H. F.; Zhu, N.; Song, L. P.; Deng, H. M. Chin. J. Org. Chem. 2015, 35, 2595 (in Chinese). (徐姗姗, 张敏, 张丽, 蒋海芳, 朱宁, 宋力平, 邓红梅, 有机化 学, 2015, 35, 2595.)

[35] He, C.; He, Q.; Chen, Q.; Shi, L.; Cao, H.; Cheng, J. Tetrahedron Lett. 2010, 51, 1317.

[36] Hao, Z. S.; Li, M. J.; Lin, H. X.; Gu, Z. B.; Cui, Y. M. Dyes Pigm. 2014, 109, 54.

[37] Budzik, B. W.; Evans, K. A.; Wisnoski, D. D.; Jin, J.; Rivero, R. A.; Szewczyk, G. R.; Jayawickreme, C.; Moncol, D. L.; Yu, H. S. Bioorg. Med. Chem. Lett. 2010, 20, 1363.

[38] CCDC 1540659 contains the supplementary crystallographic data for this paper. These data can be obtained free of charge from The Cambridge Crystallographic Data CEntre via www.ccdc.cam.ac.uk/ data request/cif.

(Li, L.; Fan, Y.) 